\title{
The NF-кB pathway regulates heterochromatin at intronic young LINE-1 elements and hematopoietic stem cell gene expression during irradiation stress
}

Yanis Pelinski ${ }^{1}$, Donia Hidaoui ${ }^{1 *}$, François Hermetet ${ }^{*}{ }^{*}$, Anne Stolz ${ }^{1}$, M'boyba Khadija Diop ${ }^{3}$, Amir M. Chioukh ${ }^{1}$, Françoise Porteu ${ }^{1 *}$, and Emilie Elvira-Matelot ${ }^{1 *}$, .

${ }^{1}$ Inserm UMR1287, Université Paris-Saclay, Gustave Roussy, 94805, Villejuif, France.

${ }^{2}$ Bioinformatics Platform UMS AMMICa INSERM US23/CNRS 3655, Université ParisSaclay, Gustave Roussy, 94805, Villejuif, France.

*Equal contribution

$\S_{\text {to }}$ whom the correspondence should be sent:

Françoise Porteu (francoise.porteu@gustaveroussy.fr)

Emilie Elvira-Matelot (emilie.elvira-matelot@inserm.fr)

INSERM U1287

Cancer Campus Gustave Roussy, PR1

114 rue Édouard-Vaillant

94805 Villejuif Cedex-France

Tel: (33) 142112348 


\section{SUMMARY}

Understanding how ionizing radiations (IR) alter hematopoietic stem cell (HSC) function on the long-term is crucial. We recently showed a link between derepression of L1Md, the mouse young subfamilies of LINE-1/L1 retroelements, and IR-induced HSC injury. L1 contribute to gene regulatory networks. However, the mechanisms involved in IR-induced L1Md derepression, and their impact on HSC transcriptome remain to be addressed. Here we show that IR triggers genome-wide $\mathrm{H} 3 \mathrm{~K} 9 \mathrm{me} 3$ decreased and transcriptomic changes in HSCs, characterized by a loss of the TNF- $\alpha / \mathrm{NF}-\kappa \mathrm{B}$ and HSC signatures. HSC gene repression is associated to $\mathrm{H} 3 \mathrm{~K} 9 \mathrm{me} 3$ loss at specific intronic L1Md displaying NF-KB binding sites. This is correlated with reduced NFKB1 repressor expression. TNF- $\alpha$ treatment before IR rescued all these effects and prevented IR-induced HSC loss of function in vivo. This reveals the importance of the TNF- $\alpha / \mathrm{NF}-\kappa \mathrm{B}$ pathway to control $\mathrm{H} 3 \mathrm{~K} 9 \mathrm{me} 3$ levels at selected intronic L1Md and thereby preserve HSC gene expression and function during IR stress.

Keywords: hematopoietic stem cells, irradiation, heterochromatin, retroelements, TNF- $\alpha$, NF-кB. 


\section{INTRODUCTION}

Exposure to ionizing radiations (IR), in the context of medical use such as radiotherapies, is an independent risk factor for many disorders characteristic of an accelerated aging. The hematopoietic tissue is particularly sensitive to IR. Therapy-related myeloid neoplasms (t$\mathrm{MN}$ ) develop between 3 to 8 years after exposure to chemotherapies or radiotherapies, are frequently associated with poor overall survival, and are generally refractory to conventional therapies (Takahashi et al., 2018). This has become a major public concern in the last decades as the number of long-term survivors of cancer treatments is continuously growing.

HSCs self-renew and give rise to all blood cell lineages. Maintenance of their integrity is crucial throughout life. We and others have shown that total body irradiation (TBI) in mice leads to long-term defects in hematopoiesis due to loss of HSC reserves and functions (Mohrin et al., 2010; de Laval et al., 2013; Fleenor et al., 2015). In HSCs, IR induces DNA damage accumulation, loss of self-renewal, and a biased differentiation towards the myeloid lineage leading to increased myeloid cell counts and decline of the adaptive immune response. These changes are likely contributing to many IR-induced premature aging disorders and to the high risk of developing t-MN. Understanding the molecular mechanisms leading to HSC loss of function upon IR is necessary to modulate its adverse effects. It may also help identifying the first events leading to hematologic malignancies.

IR induces DNA double strand breaks (DSBs). DNA damage is thought to be one of the main driving forces of aging. However, delaying the effects of age in mice by decreasing the levels of DNA damage has never been achieved, and a direct link between DSB formation and physiological aging is still lacking (White and Vijg, 2016). Although IR has been shown to induce chromosomal abnormalities in progenitors (Mohrin et al., 2010; de Laval et al., 2013, 2014), in fact HSCs are quite resilient towards accumulating DNA mutations in response to 
DNA damage (Moehrle et al., 2015). In addition, there is no direct evidence that IR-induced DSBs are directly linked to long-lasting defects in HSC differentiation and self-renewal.

In addition to DSBs, IR has been shown to induce changes in chromatin state, mainly at the level of DNA methylation, in different tissues and cell lines (Miousse et al., 2017a). Epigenetic alterations have been observed in aged HSCs in the absence of mutations in epigenetic factors (Sun et al., 2014, Djeghloul et al., 2016). Reorganization of heterochromatin is among the most commonly-reported changes in aging and senescence supporting its essential role in maintaining proper cellular function (Tsurumi et al., 2012). Maintenance of HSC identity is dependent on the heterochromatin mark H3K9me3 (Koide et al., 2016). Decreased H3K9me3 in HSCs due to loss of Suv39h2 and/or Suv39h1 methyltransferases occurs with age and is associated with the loss of B cell differentiation (Djeghloul et al., 2016) and hematopoietic changes archetypal of aging (Djeghloul et al., 2016; Keenan et al., 2021). However, whether the long-term effects of IR on HSCs is linked to IR-induced changes in heterochromatin remains to be addressed.

Heterochromatin plays also a major role in the maintenance of the genome stability by repressing transposable elements (TEs), including DNA transposons, and retrotransposable elements (RTEs), further classified as long terminal repeat (LTR) sequences which characterize endogenous retroviruses (ERV), and non-LTR elements such as long or short interspersed elements (LINE-1/L1; SINE). Propagation of RTE in the genome has been recognized as a major source of genomic instability. Even without propagating, RTE have also been recently recognized as major contributors of gene regulatory networks (Chuong et al., 2017). Indeed, they qualitatively and quantitatively control gene expression, providing alternative enhancers, promoters, splicing or polyadenylation signals, and also serving as cisregulatory elements in a cell specific fashion. Basal L1 expression in early mouse embryo is necessary for its proper development (Jachowicz et al., 2017). RTE are involved in T-cell 
differentiation by regulating genes involved in immune processes (Adoue et al., 2019). Abnormal RTE expression has been observed in cancers, including AML, and may be involved in the pathogenesis through the alteration of host gene expression (Chuong et al., 2017) and the expression of oncogenes (Jang et al., 2019; Deniz et al., 2020).

We recently showed that evolutionary recent mouse L1 (L1Md) are highly expressed in HSCs and that their expression is further increased and maintained at high levels up to 1 month after TBI. This is linked to persistent DNA-damage (Barbieri et al., 2018). The decreased H3K9me3 in aged HSCs is also associated with increased L1Md expression (Djeghloul et al., 2016). However, the impact of L1Md derepression on the HSC transcriptome remains to be addressed. In addition the mechanisms and signaling pathways by which IR specifically triggers L1Md expression in HSCs is currently unknown. We and others previously showed that the NF-אB signaling pathway is required to prevent IR-induced HSC injury (de Laval et al., 2014; $\mathrm{Hu}$ et al., 2020). In addition, tumor necrosis factor $\alpha$ (TNF- $\alpha$ )-induced NF- $\mathrm{B}$ supports HSC survival during inflammation and chemotherapeutic stress induced by 5fluorouracil (Yamashita and Passegué, 2019). On the other hand, basal activation of NF-кB is required for HSC homeostasis and self-renewal potential and the expression of key HSC maintenance genes are severely impaired in mice deficient for NF- $\mathrm{KB}$ pathway factors (Stein and Baldwin, 2013; Fang et al., 2018; Hu et al., 2020).

We show here that IR induces a major loss of H3K9me3 in HSCs, which mainly affects L1Md subfamilies, and more specifically intronic L1Md displaying NF- $\mathrm{KB}$ binding sites. By controlling the levels of $\mathrm{H} 3 \mathrm{~K} 9 \mathrm{me} 3$ at selected intronic L1Md located in genes belonging to the HSC signature, this pathway plays a crucial role to preserve HSC specific gene expression and HSC function during IR stress. 


\section{RESULTS}

\section{H3K9me3 is mainly enriched at recent L1Md subfamilies in HSCs}

To assess the effect of IR on $\mathrm{H} 3 \mathrm{~K} 9 \mathrm{me} 3$ in HSCs and more particularly at TE sequences, we performed H3K9me3 ChIP-seq experiments in HSCs ( $\left.\mathrm{Lin}^{-} \mathrm{Sca}^{+}-\mathrm{Kit}^{+}-\mathrm{CD} 34^{-} \mathrm{Flk} 2^{-}\right)$sorted from mice one month after TBI (2Gy) or not, as previously described (Barbieri et al., 2018) (Figure 1A). Deep characterization of H3K9me3 enrichment in HSCs, notably at TE sequences, has never been performed. We thus first characterized H3K9me3 genomic coverage in HSCs at steady state.

Analysis of genomic repeats is still a bioinformatics challenge. The assignment of reads complementary to sequences that are repeated in the genome and that present low sequence variation is largely compromised. These multiple mapping reads are often discarded in ChIPseq studies. However, when using only unique mapping reads, one can induce a bias of representation of the TE subfamilies towards the oldest ones as the youngest subfamilies, such as L1Md, present a very low mappability due to quasi-identical copies (Teissandier et al., 2019). Therefore, while young families of TEs are the most epigenetically regulated in the genome (Pezic et al.et al.,, 2014; Barau et al., 2016), they are severely underestimated in unique read analysis.

To maximize the output information on these sequences, we considered all reads that mapped to the genome without mismatches and randomly assigned them at one of their best possible position in the genome (Figure 1A). We combined both unique and multiple mapping reads analyses (U-MRA and M-MRA, respectively) to finally get a compromise between precise assignment of the unique reads and global information at youngest TE subfamily level.

Quality control of the resulting reads indicated high genomic coverage in both U- and MMRA and in NIR and IR conditions (Table S1). Peak calling followed by reproducibility measurement between replicates (Irreproducible discovery rate=IDR) identified 456 peaks on 
average in the NIR conditions in the U-MRA. As expected, with 7769 peaks, M-MRA gave a substantial increased number of peaks (Figure 1B; Table S1). Our peak calling retrieved the SUV39h1 and 2-dependent H3K9me3 peaks that were previously described at young TEs such as IAP ERVs (Figure S1A) using this strategy in mouse embryonic stem cells (mESCs) (Bulut-Karslioglu et al., 2014). Of note, these elements were not covered by U-MRA.

After annotation of the peaks using annotateR, both U-MRA and M-MRA showed that the majority of the $\mathrm{H} 3 \mathrm{~K} 9 \mathrm{me} 3$-enriched peaks occurs at TEs $(41.9 \%$ and $52 \%$ respectively) (Figure 1C). Interestingly, M-MRA shows a gain in LINE representation, with $32.7 \%$ of the total $\mathrm{H} 3 \mathrm{~K} 9 \mathrm{me} 3$ enriched peaks vs $16 \%$ in U-MRA. By contrast, M-MRA shows reduced SINE and DNA representation while LTR representation was not affected.

We next precisely quantified $\mathrm{H} 3 \mathrm{~K} 9 \mathrm{me} 3$ levels from M-MRA over the different TE families using the reconstructed repeatMasker annotations (Walter et al., 2016), as described (BaillyBechet et al., 2014). We found that the majority of H3K9me3 enrichment at TEs occurs at LINEs followed by ERVs, SINEs, and DNA (Figure 1D). Normalization of H3K9me3 enrichment to the number of corresponding TE loci in the genome gave similar results and confirmed the major H3K9me3 enrichment at LINEs in HSCs (Figure S1B).

Since young TE subfamilies, notably L1Md, were previously reported to be the most epigenetically regulated in the genome compared to old LINEs in ESCs and testis (Pezic et al., 2014; Barau.et al., 2016), we further dissected H3K9me3 enrichment at TEs depending on their age, as calculated in (Sookdeo et al., 2013). We observed a significant negative correlation between the age of the LINE and H3K9me3 enrichment, with the youngest, typically L1Md_A and L1Md_Tf, showing the highest enrichment (Figure 1E), as previously observed for DNA methylation (Miousse et al., 2017b).

Altogether, these data demonstrate that $\mathrm{H} 3 \mathrm{~K} 9 \mathrm{me} 3$ is mainly enriched at repetitive sequences, and more particularly at young L1Md elements in HSCs. 


\section{Irradiation induces a loss of H3K9me3 that mainly affects the recent L1Md subfamilies}

We next investigated changes in H3K9me3 that occur upon IR. We first compared the number of $\mathrm{H} 3 \mathrm{~K} 9 \mathrm{me} 3$ peaks identified in IR vs NIR conditions. Only 115 peaks in average were found in IR in the U-MRA, compared to 456 peaks in NIR condition, and 283 compared to 7769 in the M-MRA (Figure 1B).

Differential $\mathrm{H} 3 \mathrm{~K} 9 \mathrm{me} 3$ enrichment analysis performed at $\mathrm{H} 3 \mathrm{~K} 9 \mathrm{me} 3$ peaks identified in both IR and NIR conditions further revealed a strong decrease in $\mathrm{H} 3 \mathrm{~K} 9 \mathrm{me} 3$ enrichment. We found 13 and 253 peaks showing significant $(p<0.05)$ differential H3K9me3 enrichment upon IR in U- and M-MRA, respectively. All of them in U-MRA and 252/253 peaks in M-MRA showed decreased H3K9me3 upon IR (Figure 2A). These data reveal a major loss of H3K9me3 genomic coverage upon IR, regarding both the number of peaks and the concentration of H3K9me3 at conserved peaks.

U-MRA peak annotation in IR condition revealed a loss of representation at LINEs, promoters and 5'UTR regions and a gain at SINEs (Figure 1C). M-MRA further confirmed the loss of representation at LINE families (Figure 1C). H3K9me3 quantification at TEs further confirmed that loss of H3K9me3 mainly affect LINE compared to other TE families, which is counterbalanced by a gain in representation of SINE (Figure 1D). No change was observed for LTR in either peak annotation or H3K9me3 quantification. Heatmap representation of the average concentration of $\mathrm{H} 3 \mathrm{~K} 9 \mathrm{me} 3$ at peaks retrieved from M-MRA further confirmed the strong H3K9me3 loss upon IR affecting mainly LINEs as compared to LTR, and even more as compared to SINEs or DNA. The decrease in H3K9me3 induced by IR is particularly striking at young LINEs such as L1Md, compared to older Lx5 subfamilies (Figure 2B). Further quantification of $\mathrm{H} 3 \mathrm{~K} 9 \mathrm{me} 3$ concentration enrichment over the different families of elements depending on their age (Sookdeo et al., 2013) confirmed that youngest 
LINEs are the TE families that are the most affected by IR, as compared to LTR, SINEs and DNA (Figure S1C).

Altogether these data reveal that one month after IR, HSCs display a major loss of H3K9me3, which mainly occurs at L1Md, the subfamilies of TEs the most enriched in H3K9me3 at steady state. This prompted us to further focus our analysis on L1Md.

Plot profile of $\mathrm{H} 3 \mathrm{~K} 9 \mathrm{me} 3$ enrichment along L1Md sequences showed asymmetric distribution of H3K9me3 along the L1 body, with the highest enrichment and the highest loss upon IR at the 5' end of these elements (Figures S1D). L1 retrotransposition is often abortive and results in the insertion of copies truncated in their 5' end. Thus, a majority of L1 does not harbor the 5' end. Plot profile centered on the longest L1Md ( $\geq 5 \mathrm{~kb})$ enriched in L1 harboring promoter sequences further confirms that IR mainly decreases H3K9me3 levels at this location (Figure 2C). Finally, we confirmed the global decrease of $\mathrm{H} 3 \mathrm{~K} 9 \mathrm{me} 3$ at L1Md promoters, and more particularly at L1Md_A, using ChIP-qPCR experiments with primers recognizing either all L1Md or specifically L1Md_A promoter (Figure 2D), as described previously (Barbieri et al., 2018; Djeghloul et al., 2016).

\section{Irradiation induces a strong deregulation of the HSC transcriptome}

In order to unravel the consequences of IR-induced $\mathrm{H} 3 \mathrm{~K} 9 \mathrm{me} 3$ loss on the HSC transcriptome, we performed RNA-seq of HSCs one month after TBI (Figure 1A). Comparison of IR vs NIR revealed 1067 differentially expressed genes (DEGs) $(p<0.05)$, with 602 (56.4\%) genes downregulated and 465 (43\%) genes upregulated upon IR (Figures 3A and 3B; Table S2). Differences in gene expression are very strong, as almost $80 \%$ of the DEGs between IR and NIR present a fold change above 10 and more than $50 \%$ present a fold change above 50 (Figure 3B). Gene Set enrichment analysis (GSEA) on Hallmark gene sets indicated significant enrichments in DNA repair, G2/M checkpoint and oxidative phosphorylation pathways, as expected upon IR (Figure 3C), whereas the main pathways lost in IR are related 
to cell signaling (Figure 3D). Among these, the most significant decrease concerns genes regulated by NF-KB in response to TNF- $\alpha$ (Figures 3D and 3E). A recent report showed that TNF- $\alpha$ induces a specific prosurvival gene signature in HSCs (Yamashita and Passegué, 2019). Interrogating this gene signature, composed of 62 genes representing both core regulators of the NF-kB pathway and TNF $\alpha$-induced HSC-specific survival genes, we observed its significant loss upon IR (Figure 3F). IR also induced the loss of the different HSC TNF- $\alpha$ signatures taken individually: the two in vitro signatures obtained $3 \mathrm{~h}$ and $12 \mathrm{~h}$ after $\mathrm{TNF} \alpha$ treatment and the in vivo signature obtained $3 \mathrm{~h}$ after $\mathrm{TNF} \alpha$ treatment in mice (Figure S2A). By contrast, IR had no effect on the GMP TNF- $\alpha$ gene signature (Figure 3G) (Yamashita and Passegué, 2019).

Given the importance of the NF-кB pathway at maintaining HSC survival and self-renewal or during chemotherapeutic and IR stress (de Laval et al., 2014; Yamashita and Passegué, 2019; $\mathrm{Hu}$ et al., 2020), we next interrogated different published HSC signatures that are enriched in low-output/self-renewing and functional long-term regeneration HSCs compared to differentiating HSCs, or in dormant vs activated HSCs (Chambers et al., 2008; Pietras et al., 2015; Cabezas-Wallscheid et al., 2017; Rodriguez-Fraticelli et al., 2020). We found a significant loss of all these signatures upon IR (Figures 3H, Figure S2B). Likewise, the megakaryocyte (MK)-Biased output HSC signature (Rodriguez-Fraticelli et al., 2020), representing platelet-primed HSCs that were also previously described to be at the apex of the HSC hierarchy (Sanjuan-Pla et al., 2013), was also enriched in NIR vs IR HSCs (Figure S2B). Conversely, the high-output and multilineage signatures (Rodriguez-Fraticelli et al.,, 2020), which mark differentiating HSCs, showed a significant enrichment upon IR (Figure S2C). Altogether, these data show that IR induces a loss of transcriptional signatures involved in HSC quiescence, long-term potency and self-renewal capacity, and a gain in gene 
signatures involved in HSC differentiation, thus recapitulating the HSC loss of self-renewal previously reported upon IR (de Laval et al., 2013).

\section{IR-induced downregulation of gene expression is associated with the presence of L1Md} in their introns

U-MRA of H3K9me3 enrichment showed a loss of promoter representation upon IR (Figure 1C), suggesting decreased $\mathrm{H} 3 \mathrm{~K} 9 \mathrm{me} 3$ level at promoter sequences. Since this could be associated with gene deregulation, particularly gene upregulation, we quantified H3K9me3 enrichment over promoter regions $(-2 \mathrm{~kb} ;+1 \mathrm{~kb}$ around TSS). We found 239 promoter sequences showing a significant $(p<0.05) \mathrm{H} 3 \mathrm{~K} 9 \mathrm{me} 3$ differential enrichment upon IR, 211 and 28 showing decreased and increased H3K9me3, respectively (Figure S3A). However, these variations are not correlated with gene upregulation, and only very poorly correlated with gene downregulation (Figures 4A and S3B).

Since the loss of H3K9me3 upon IR mainly occurs at L1Md, we sought to determine if L1Md derepression might be involved in the gene deregulation observed after IR. To address this question, we crossed the list of the 1067 DEGs $(p<0.05)$ in IR $v s$ NIR from our RNA-seq data with the list of genes hosting one or several L1Md (reconstructed repeatMasker database) (Figure 4B; Table S3). The vast majority of these L1Md are located in introns (99\%) (Figure S3C). We found that 377 DEGs in IR host one or several L1Md, in majority located in introns. This is significantly $(p<0.0001)$ more that one would expect by chance, as revealed by a permutation test using 10,000 lists of 1067 genes randomly extracted from the refseq database (Figures 4B and 4C). These results reveal a strong and significant association between gene deregulation upon IR and the presence of an intronic L1Md in these genes. This is specific for L1Md as no significant association was observed between DEGs and the presence of Lx5, an older LINE subfamily (Figure 4D). Surprisingly, this association is 
specific for genes that are downregulated upon IR (Figure 4E) and was not found for upregulated genes (Figure 4F; Table S3).

Interestingly, $50 \%$ of the genes from LT-HSC signatures (Chambers et al., 2007; Pietras et al., 2015) whose expression is significantly decreased upon IR (Figures $4 \mathbf{H}$ and 4I) contain one or several L1Md in their introns. Similarly, we found that $31 \%$ and $41 \%$ of the genes from the low-output and MK-biased signatures (Rodriguez-Fraticelli et al., 2020) host one or several L1Md (Figures S3D and S3E). Altogether, these data highlight a significant association between genes whose expression is repressed upon IR, notably those belonging to the HSC signature, and the presence of intronic L1Md.

\section{Gene repression upon IR is associated with loss of $\mathrm{H3K} 9 \mathrm{me} 3$ at selected intronic L1Md loci harboring NF-KB binding sites}

We next investigated if the loss of $\mathrm{H} 3 \mathrm{~K} 9 \mathrm{me} 3$ induced by IR at intronic L1Md in a given gene could be associated with its decreased expression in cis. For this purpose we chose 3 HSC candidate genes whose expression is significantly reduced by IR in our RNA-seq data: Mecom, Pkia and Ttc8 (Table S2), as well as 3 negative controls, also harboring at least one intronic L1Md but whose expression remains unchanged upon $\operatorname{IR}(0.7<\mathrm{FC}<1.3, \mathrm{p}>0.05)$ : Snx27, Mapre2, and Celf2. One month after TBI, a significant downregulation of Mecom, Pkia and Ttc8, but not of the 3 controls was observed (Figures 5A and 5B). To assess H3K9me3 enrichment specifically at intronic L1Md of these genes, we performed H3K9me3 ChIP-qPCR experiments using primer pairs located both in the intron and in the 5 ' end of the L1Md, thus allowing unique and specific amplicon production (Figure 5C). We chose in each case the longest L1Md ( $>5 \mathrm{~kb})$, displaying higher enrichment at the 5' end (Figure 2C), to detect significant basal $\mathrm{H} 3 \mathrm{~K} 9 \mathrm{me} 3$. We first confirmed that $\mathrm{H} 3 \mathrm{~K} 9 \mathrm{me} 3$ is indeed present at all intronic L1Md, as compared to Spil and 5S negative controls (Figures S4A and S4B). Of 
note, H3K9me3 levels at all these L1Md are similar to that found globally at L1Md_A promoters (Figure S4C). IR induced a significant decrease in H3K9me3 specifically at all the chosen canditate intronic L1Md of genes downregulated upon IR, but not at the control genes (Figure 5D). This indicates that IR-induced H3K9me3 loss at intronic L1Md is not a general event but rather that it seems to occur only in specific genes whose expression is reduced upon IR. This suggests that the presence of the H3K9me3 mark at this location may play a role in the regulation of gene expression.

In order to unravel what makes the specificity of H3K9me3 loss at L1Md upon IR, we interrogated the potential enrichment for binding motifs for transcription factors in the L1Md located in genes downregulated in IR $(p<0.05,1069 \mathrm{~L} 1 \mathrm{Md}=$ input sequences $)$ vs nonderegulated genes $(p>0.05,0.7<\mathrm{FC}<1.3,1310 \mathrm{~L} 1 \mathrm{Md}=$ background sequences $)$ through de novo motif search using BAMMmotif (https://bammmotif.soedinglab.org/) (Kiesel et al., 2018). Of the 4 motifs found significantly enriched in L1Md located in downregulated genes, 2 were not retrieved in the mouse Hocomoco motif database, one motif corresponds to the binding site of Hoxal, and one to the binding site of Rel, a member of the NF-KB transcription factor family (Figure 5E). De novo motif search in the $\mathrm{L} 1 \mathrm{Md}$ located in downregulated genes $v s$ upregulated genes also retrieved Rel motif (Figure S5A). Furthermore, de novo search for motifs specifically enriched in L1Md located in genes participating (284 L1Md) vs genes not participating (793 L1Md) to the loss of the HSC signature (Figure 3H) also revealed significant enrichment for NF- $\mathrm{KB}$ transcription factors Rel, RelA and Nfkb1 (Figure 5F). Similar results were found for the low-ouput / selfrenewing LT-HSC signatures (Figures S4B and S4C).

Of note, this motif is present in the intronic L1Md of IR-regulated genes Mecom, Pkia and Ttc8 which loose H3K9me3 upon IR (Figure 5D, Table S3), supporting the possibility that it may regulate the presence of $\mathrm{H} 3 \mathrm{~K} 9 \mathrm{me} 3$ at these loci. 
Performing de novo search on promoter sequences of genes downregulated by $\operatorname{IR}(\mathrm{p}<0.05$, 3893 promoter sequences) $v s$ non-deregulated genes $(0.7<\mathrm{FC}<1.3, \mathrm{p}>0.05,14208$ promoter sequences from which we randomly extracted 3893 sequences) did not show specific enrichment in motifs for NF- $\kappa \mathrm{B}$ members. Instead, motifs for different transcription factors such as Egr, Znf, Foxj, Foxq were found (Figure S4D).

Altogether, these data suggest that the NF-кB pathway may control HSC gene expression by regulating the presence of the $\mathrm{H} 3 \mathrm{~K} 9 \mathrm{me} 3$ mark at L1Md located in introns, and not by affecting promoters.

TNF $\alpha$ treatment prevents loss of $\mathrm{H3H9me3}$ at intronic L1Md, HSC gene repression and

\section{HSC loss of function during IR stress}

In mammals, the NF-кB family is composed of five members: RELA (p65), RELB, c-REL, and the precursor proteins NFKB1 (p105) and NFKB2 (p100), which are processed into their active forms, p50 and p52 respectively, and are active as homo or heterodimers (Cartwright et al., 2016). The canonical NF-кB pathway involves p50, p65 and c-Rel. P50 lacks transactivation domain. Thus, while p50/p65 or c-Rel heterodimers act as transcriptional activators, p50/p50 (NFKB1) homodimers are generally described as transcriptional repressors. NFKB1 notably represses the expression of proinflammatory genes through the recruitment of chromatin modifiers and H3K9 methylation (Elsharkawy et al., 2010; Ea et al., 2012). In addition, p50 has been shown to shuttle between the nucleus and cytoplasm and to bind to a large number of genes in unstimulated cells (Schreiber et al., 2006). This makes of

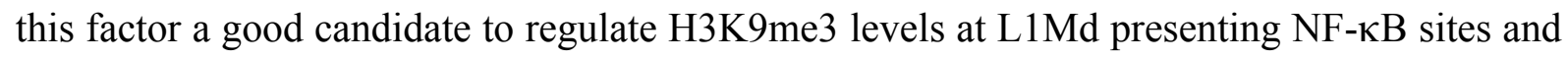
IR-induced HSC gene expression changes. Supporting this possibility, HSCs sorted from mice one month after TBI showed a significant decrease in both NFKB1 mRNA and protein expression (Figures 6A-C). Processing of p105 to p50 is regulated both independently of the 
$\mathrm{NF}-\kappa \mathrm{B}$ activation pathway and during activation of the canonical pathway induced by proinflammatory cytokines such as TNF- $\alpha$ (Cartwright et al., 2016). As shown above, IR induces a loss of the TNFA_signaling_Via_NFKB signature (Figures 3D and 3E). Thus, we asked whether TNF- $\alpha$ stimulation could prevent IR effects by rescuing the levels of p50 homodimers. NFKB1 protein expression decreased after $48 \mathrm{~h}$ of culture of purified HSCs that have been irradiated in vitro (Figures 6D and 6E). Addition of TNF- $\alpha$ to the cell medium prior to IR prevented this effect, and even increased NFKB1 expression when compared to the non-stimulated condition. As after TBI, the loss of NFKB1 induced by IR in vitro was associated with a specific decrease of Mecom, Pkia and Ttc8 but not of Celf2, Snx27 and Mapre 2 mRNAs (Figures 6F and S6). Importantly, H3K9me3 ChIP-qPCR at intronic L1Md of the selected genes correlated with gene expression with a significant and specific reduction at Mecom and Pkia intronic L1Md but not at Snx27 and Celf2 (Figure 6G). This shows that IR-induced H3K9me3 loss at intronic L1Md and gene expression changes in HSCs are direct and short-term and that TNF- $\alpha$ stimulation can rescue both effects.

Finally, to confirm the importance of this pathway in HSC maintenance, we investigated the effect of TNF- $\alpha$ treatment on HSC function upon IR in vivo. Total BM cells isolated from mice treated with TNF- $\alpha$ lh before TBI or not were transplanted in competition with total BM cells from mice ubiquitously expressing GFP (ubi-GFP mice) into lethally irradiated ubiGFP mice (Schaefer et al., 2001) (Figure 6H). Three months after reconstitution, as expected, the percentage of GFP- IR donnor cells in the blood was greatly decreased. TNF- $\alpha$ treatment before TBI significantly prevented this effect (Figures 6I and 6J). It also significantly prevented IR-induced LT-HSC loss (Figures 6K and 6L).

Altogether, these data suggest that TNF- $\alpha$ treatment rescues HSC reconstitution ability upon IR by preventing IR-induced decrease in NFKB1 repressor expression, specific derepression 
bioRxiv preprint doi: https://doi.org/10.1101/2021.06.08.447574; this version posted June 8, 2021. The copyright holder for this preprint (which was not certified by peer review) is the author/funder. All rights reserved. No reuse allowed without permission.

of L1Md harboring NF-kB binding sites in the introns of HSC genes, and thereby their repression (Figure 6M). 


\section{DISCUSSION}

Epigenetic factors controlling DNA and histone methylation are key regulators of HSC function and are often mutated in leukemia. These mutations also increase with age in otherwise healthy individuals where they confer clonal hematopoiesis. However, epigenetic alterations have been observed in aged HSCs in the absence of mutations in epigenetic factors (Sun et al., 2014; Djeghloul et al., 2016). HSC aging phenotype is reversible, suggesting that it results from an altered epigenome (Wahlestedt et al., 2017) that may predispose to the development of leukemia (Adelman et al., 2019).

$\mathrm{H} 3 \mathrm{~K} 9 \mathrm{me} 3$ alterations is a hallmark of aging and cellular senescence in model organisms (Criscione et al., 2016; Ocampo et al., 2016). Although H3K9me3 has been shown to be crucial for HSC identity (Koide et al., 2016) and have been linked to leukemogenesis (Monaghan et al., 2019), H3K9me3 changes in HSCs have, to our knowledge, never been studied in the context of stress such as IR. We show here that IR stress profoundly affect HSC heterochromatin by significantly reducing $\mathrm{H} 3 \mathrm{~K} 9 \mathrm{me} 3$ enrichment and that this loss mainly occurs at L1Md elements. This effect was observed both after short time in vitro and long time after TBI in vivo, suggesting that heterochromatin alterations may explain the long-term effect of IR on HSC function.

Heterochromatin alterations are associated with a strong deregulation of the HSC transcriptome. $\mathrm{H} 3 \mathrm{~K} 9 \mathrm{me} 3$ enrichment at promoters has recently emerged as a key player in the repression of lineage-inappropriate genes (Koide et al., 2016). In AML, these changes have been shown to correlate with patient prognosis (Müller-Tidow et al., 2010). Surprisingly, we found here that gene deregulation is not associated with H3K9me3 changes at gene promoters, but is rather associated with the loss of $\mathrm{H} 3 \mathrm{~K} 9 \mathrm{me} 3$ at intronic L1Md. H3K9me3 enriched at intronic evolutionary recent L1Md was previously shown to be involved in the tight regulation of gene transcription in ESCs (Liu et al., 2018). Some ERVs also play the role 
of AML enhancers with a driving role in leukemia cell phenotype (Deniz et al., 2020). However, our study is the first showing the involvement of L1Md on the regulation of HSC gene expression.

Although surprising at first glance, repression of genes following derepression of intragenic L1 was previously reported in cancers (Aporntewan et al., 2011). This may be due to transcriptional interference (Han et al., 2004; Kaer et al., 2011; Ninova et al., 2020), mostly through intron retention, exonization and cryptic polyAdenylation (Kaer et al., 2011). H3K9me3 is mostly enriched in the 5'UTR part of the L1Md. Loss of this repressive mark at the $\mathrm{L} 1 \mathrm{Md}$ promoter regions may induce its transcription and gene repression through transcriptional interference. We cannot test directly this hypothesis due to the repetitive nature of these sequences. However, gene repression is associated with the presence of L1Md in their introns whatever their size, and after IR, we could observe downregulated genes losing H3K9me3 enrichment at intronic L1Md lacking 5'UTR sequences (data not shown). Thus, although we cannot rule out the possibility of a transcription arising from the 3'part of the L1, as previously suggested (Faulkner et al., 2009), these data suggest that gene repression may not be exclusively due to intronic L1Md expression.

Instead of blocking transcription, DNA methylation or H3K9me3 within gene bodies is a feature of transcribed gene (Vakoc et al., 2005; Jones, 2012; Ninova et al., 2020). H3K9me3 loss in gene bodies was previously shown to be associated with gene repression (Ninova et al., 2020). The presence of H3K9me3 islands in the body of the genes has been proposed to slow-down RNA Polymerase II (RNAP II) elongation rate (Vakoc et al., 2005; Saint-andré et al., 2011). This was shown to help the recognition of true $v s$ cryptic RNA processing sites, controlling alternative splicing (De La Mata et al., 2003), polyadenylation and finally transcript stability. This is particularly relevant in the case of genes bearing long introns. Interestingly, the genes downregulated upon IR in HSCs are significantly longer than by 
chance (data not shown). Slowing down RNAP II elongation rate in these long intron genes might also prevent R-loop formation and genomic instability (Aguilera and Gaillard, 2014). Interestingly, evolutionary young L1 located in long introns recruit RNA-binding proteins to prevent improper splicing or polyAdenylation (Attig et al., 2018). Thus, even in the absence of intronic L1Md transcription, loss of H3K9me3 islands enriched at intronic L1Md in the body of the genes might be involved in splicing defects and/or premature polyadenylation. Whether these mechanisms are involved in HSC gene regulation will require further investigations.

Our RNA-seq analysis indicates a strong downregulation of the TNF- $\alpha-N F-\kappa B$ gene signature, including one month after TBI. IR specifically reduced the recently described HSC-prosurvival TNF $\alpha-N F-\kappa B$ signature required to maintain HSCs during inflammation or cytotoxic BM ablation (Yamashita and Passegué, 2019). This suggests that loss of long-term regenerating HSC and TNF- $\alpha-\mathrm{NF}-\kappa \mathrm{B}$ gene expression upon IR may be linked. Supporting this possibility, treatment of HSCs with TNF- $\alpha$ before IR in vitro, or its injection to mice before TBI, restored HSC gene expression and their reconstitution ability. These results strongly support previous data showing that TNF- $\alpha-N F-\kappa B$ signaling is required to regulate HSC function under stress (Laval et al., 2014; Yamashita and Passegué, 2019; Hu et al., 2020). TNF- $\alpha$ promotes HSC survival through p65/RelA NF-кB subunit (Yamashita and Passegué, 2019). This factor has also been found to control the expression of genes involved in HSC maintenance (Stein and Baldwin, 2013). However, the promoters of HSC genes downregulated upon IR are not enriched in NF-кB binding sites. We show that L1Md

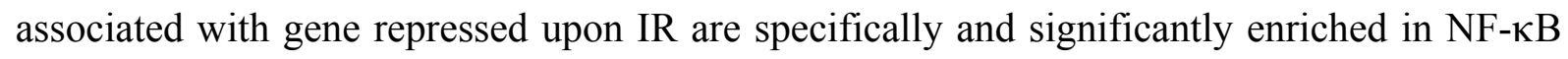
binding sites, and that this pathway regulates gene expression by controlling the level of $\mathrm{H} 3 \mathrm{~K} 9 \mathrm{me} 3$ at these sequences. Interestingly, $\mathrm{H} 3 \mathrm{~K} 9 \mathrm{me} 3$ enriched genomic regions specific to 


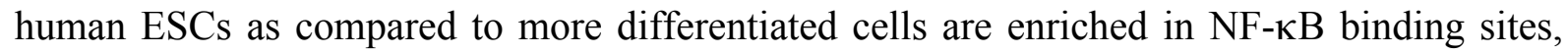
suggesting their importance in establishing and maintaining the pluripotent state (Whitaker et al., 2015).

Whereas most of the NF- $\mathrm{KB}$ members can form active transcription factors, NFKB1 p50 subunit lacks transactivation domain and p50:p50 homodimers have been shown to act as stimulus-specific repressors notably during the resolution phase of inflammation, by recruiting $\mathrm{H} 3 \mathrm{~K} 9$ methyltransferases and $\mathrm{HDAC}$ at both $\mathrm{NF}-\mathrm{\kappa B}$ and type-I interferon (IFN) response genes (Elsharkawy et al., 2010; Ea et al., 2012; Cartwright et al., 2018). p50:p65 heterodimers are the most abundant form of NF-кB generated upon inflammatory stimuli. By contrast, p50 homodimers predominate in unstimulated cells where they can be prebound to the chromatin (Schreiber et al., 2006; Cartwright et al., 2016) suggesting that this factor may also play a role under non-inflammatory conditions. Indeed, we found that NFKB1 is present in both the cytoplasm and the nucleus of resting HSCs. IR-induced downregulation of p50/NFKB1 gene and protein correlated with specific decrease in gene expression and H3K9me3 levels at intronic L1Md harboring an NF- $\mathrm{KB}$ binding site. Conversely, increasing p50 production upon TNF stimulation rescued H3K9me3 levels at the intronic L1Md and gene expression. This supports the possibility that p50:p50 homodimers might promote the enrichment of H3K9me3 at L1Md located in HSC genes and the cis-regulation of the host gene.

A growing body of evidence indicates that TEs have been coopted for transcriptional regulation in different cell and tissue types (Chuong et al., 2017). TEs are reservoirs of functional transcription factor binding sites. Since these sequence are widespread in the genome, they are largely contributing to the innovation of regulatory networks in a tissuespecific fashion (Chuong et al., 2017; Sundaram and Wang, 2018; Hermant and Torres- 
Padilla, 2021). Although LTR dominate this relationship, search for binding motifs in young L1 in human and mouse has revealed the presence of various TF motifs, including CTCF, YY1 and MYC (Sun et al., 2018; Sundaram and Wang, 2018). Our results show that NF-кB motifs are specifically enriched in most of the intronic L1Md sequences of genes downregulated during IR stress, and involve as much as $96 \%$ of these genes (Table S3). The presence of NF- $\mathrm{KB}$ binding sites in TEs is reminiscent of a study reporting that, in the human genome, $11 \%$ of NF-кB-binding sites reside in specific Alu SINEs, and that the vast majority of sites bound by NF- $\mathrm{KB}$ do not correlate with changes in gene expression (Antonaki et al., 2011). Although it is not known how many of these NF-кB motifs present in intronic L1Md have a functional role, the ability of TNF- $\alpha$ to restore both $\mathrm{H} 3 \mathrm{~K} 9 \mathrm{me} 3$ levels at the L1Md and


strongly suggests that at least some of these NF-KB-TE associations can influence gene expression.

TEs have rewired the antiviral gene regulatory network and they have been shown to play a key role in the regulatory evolution of immune response. Strong but opposing forces are driving the coevolution of TEs and antiviral defence (Chuong et al., 2016; Moelling and Broecker, 2019). Many IFN / NF-кB-target genes are viral restriction factors and contribute to the immune control of both endogenous (i.e. TEs) and exogenous genomic parasites (Schneider et al., 2014; Gazquez-Gutierrez et al., 2021). We and others have previously shown that IFN-I signaling controls young L1Md expression and L1 retrotransposition in HSCs and various tissues (Goodier et al., 2015; Yu et al., 2015; Barbieri et al., 2018). However, through the formation of dsRNA or cytoplasmic cDNA resembling viral nucleic acids, TEs are sensed by the cells as invading viruses and promote the activation of IRF3 and $\mathrm{NF}-\kappa \mathrm{B}$ transcription factors and the major antiviral immune pathways (Volkman and Stetson, 2014; Gazquez-Gutierrez et al., 2021). Notably, TE-derived dsRNAs have been shown to 
provide the inflammatory signal necessary for HSC generation during embryonic development (Lefkopoulos et al., 2020). Intringinly, beside HSC maintenance genes, many genes involved in IFN and NF-אB immune response pathway are found among genes downregulated in IR presenting an intronic L1Md with a NF-אB binding site (Table S3). These include IFN and NF-кB target genes known to control L1 retrotransposition and / or levels such as EiF2ak2 (Interferon-Inducible protein kinase R) and Oas $1 g$ RNase L whose activity are triggered by virus- or TE-derived dsRNAs; Jak2, Tyk2, Tnfrsf9 and Birc2 involved in IFN and TNF responses, respectively, as well as T cell suppressing activity genes, CD274 (PD-L1) and CD86. This further reinforces the causal relationships between TEs and immune genes and their coevolution. Interestingly, a higher TE occurrence has been found in immune gene-associated genomic regions and young TEs are specifically enriched in blood cells, as compared to other tissues (Trizzino et al., 2018; Ye et al., 2020). Enhancers unique for immune tissues are more prone to TE cooption, as compared to enhancers specific to other tissue types and the majority of them is conserved in HSC accessible chromatin regions. Using BAMMmotif for de novo motif search, we have found that the NF- $\mathrm{B}$ motif is specifically enriched in L1Md that are present in genes of the HSC signature, and in the myeloid-leucocyte-mediated-immunity signature (GO:0002444) as compared to genes enriched in pancreas, testis, kidney, liver, placenta, salivary gland (Su et al., 2002), or in genes from the immune system process ( $\mathrm{GO}: 0002376)$ as compared to genes from the

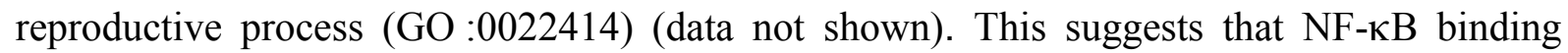
sites in L1Md might have been actively selected in introns of key HSC genes because of the immune-linked maintenance. This regulation might be important to expand the NF- $\mathrm{KB}$ and TNF- $\alpha$ activity by engaging more genes, including HSC maintenance genes into the NF- $\mathrm{KB}$ regulatory networks. Such activity could be important to maintain of HSCs while allowing

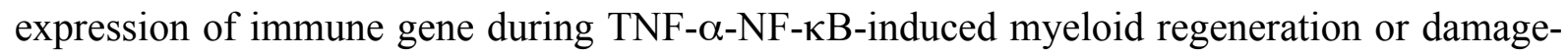


induced bone marrow ablation, and further highlight the complex role of inflammationinduced pathways in HSCs.

TEs are commonly recognized as tumor promoters (Jang et al., 2019; Grundy et al., 2021). However, by their capacity to induce DNA damage and activate cell cycle arrest, senescence and inflammatory responses, these sequences may also be viewed as tumor suppressors (Grundy et al., 2021; Kelsey, 2021). TE expression, and notably that of active full-length L1 capable of retrotranspositoin (L1Hs) is decreased in AML and MDS samples as compared to healthy $\mathrm{CD}^{+}{ }^{+}$progenitor cells (Kazachenka et al., 2019; Gu et al., 2021). In AML, oncogenic mutations leading to L1 epigenetic silencing are selected and associate with poorer prognosis ( $\mathrm{Gu}$ et al., 2021). Likewise, increased expression of the H3K9me3 methyltransferase SETDB1 is responsible for TE silencing in AML cell lines and is required for their survival (Cuellar et al., 2017). TNF- $\alpha$ levels are increased in patients with hematopoietic malignancies and the HSC-specific TNF- $\alpha$ signature is upregulated in MDS/AML malignant HSCs (Yamashita and Passegué, 2019). Exploring the mechanisms controlling TE expression and how inflammatory signals and aging impact them in normal and malignant HSC could lead to the identification of new selective dependencies of AML and new treatment strategies. 


\section{METHODS}

\section{Mice strains and treatments}

Wild type (WT) C57BL/6J mice (6-8 week-old) were from the Envigo Laboratories. All the mice were housed in a specific pathogen-free environment. All procedures were reviewed and approved by the Animal Care Committee (CE \#2019_078_23286). Mice were injected retroorbitally with $2 \mu \mathrm{g}$ TNF- $\alpha$ (Biolegend-Ozyme) before sublethal TBI (2 Gy) (RX irradiator XRAD 320).

\section{Cell harvest and culture}

Bone marrow was harvested from femur, tibia and hip bones in mice. Total bone marrow was depleted of differentiated hematopoietic cells (lineage-positive cells) using Mouse Hematopoietic Progenitor (Stem) Cell Enrichment Set (BD). Magnetically sorted Lineagenegative (lin $\left.{ }^{-}\right)$cells were kept overnight at $4^{\circ} \mathrm{C}$ in IMDM medium supplemented with $10 \%$ FBS (HyClone) and 1\% penicillin-streptomycin (Thermofisher). Staining was performed for 20min at room temperature (RT) using CD3e (Lin) - APC clone 145-2C11 (553066, BD), TER-119 (Lin) - APC clone Ter-119 (557909, BD), CD45R/B220 (Lin) - APC clone RA36B2 (553092, BD), Ly6G-6C (Lin)-APC clone RB6-8C5 (553129, BD), Ly-6A/E (Sca-1) PeCy7 clone d7 (558162, BD), CD117 (c-Kit) - PE or PerCP-Cy5.5, clone 2B8 (553355 or 560557 respectively, BD), CD34 - FITC clone RAM34 (560238, BD), CD135 (F1k2) BV421 or PE clone A2F10.1 (562898 or 553842 respectively, BD). HSCs (Lin`Sca ${ }^{+}$c$\mathrm{Kit}^{+} \mathrm{CD} 34^{\text {low }} \mathrm{Flk2} 2^{-}$) were sorted using ARIA3, ARIA Fusion or Influx cell sorters (BD Franklin Lakes, NJ, USA) and collected in Stem Span (StemCell). 
When the cells were irradiated in vitro, HSCs were cultured in medium containing Flt3Ligand, IL-3, IL-6, SCF, as described (de Laval et al., 2013) in the presence or absence of TNF- $\alpha$. TNF- $\alpha$ was added to the medium at $1 \mu \mathrm{g} / \mathrm{ml} 1$ hour before IR.

\section{Quantitative RT-PCR}

HSCs were lysed in Tri-Reagent (Zymo Research) and stored at $-80^{\circ} \mathrm{C}$ until used. Total RNA was extracted using the Direct-Zol RNA microprep kit (Zymo research) and reversetranscribed with EZ Dnase VILO (Invitrogen). Real-time PCR was performed using the SYBR pPCR premix Ex Taq (Takara) or LUNA Universal qPCR Master Mix (NEB) on a 7500 real-time PCR machine (Applied Biosystems). Samples were tested for qPCR before reverse transcription to rule out detection of contaminating DNA. qPCR primers used were designed in different exons so as to minimize possible gDNA amplification. All data were normalized to the mean expression of RPL32, and HPRT. Primer sequences are shown in Table S4.

When necessary, $1.25 \mu 1$ of cDNA was preamplified for 14 PCR cycles in a multiplex reaction

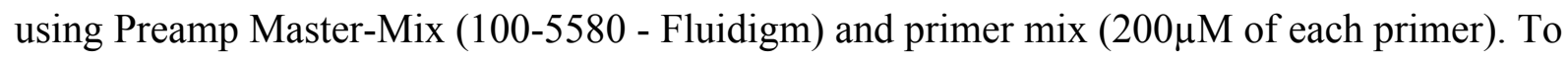
rule out primer dimerization or hairpin formation in the preamplification mix, primer sequences were previously analyzed using MFE3.0 PCR Primer Quality Control Software (Wang et al., 2019).

\section{ChIP-qPCR}

10,000 HSCs were harvested in 1ml IMDM medium supplemented with 10\% FBS and crosslinked using 1\% formaldehyde (Invitrogen) for $10 \mathrm{~min}$ at RT. ChIP-qPCR experiments were performed using the True Micro-ChIP Kit (Diagenode) according to manufacturer's instructions. Cells were sonicated using the Bioruptor Pico (Diagenode) sonication device for 
10 cycles $\left(20 \mathrm{~s}\right.$ ON/40s OFF). Chromatin was incubated overnight at $4^{\circ} \mathrm{C}$ using $0.25 \mu \mathrm{g}$ of H3K9me3 (C15410193-Diagenode) per IP. ChIP DNA was eluted and purified using the MicroChIP Diapure Columns (Diagenode). Subsequent qPCR Real-time PCR was performed as above ChIP-qPCR primers for intronic L1Md were designed such that one primer is located in the 5' region of the $\mathrm{L} 1 \mathrm{Md}$, and the other primer is located in the intron of the host gene to allow the amplification of unique and specific product (Table S4).

\section{Immunofluorescence}

3000-5000 HSCs were cytospun on glass slides and immunofluorescence was performed as previously described (de Laval et al., 2013). Monoclonal anti-NFKB1 (p50) antibody (clone E10) was purchased from Santa Cruz Biotechnology. Detection was performed using Alexa Fluor 555-coupled anti-mouse secondary antibody. All slides were visualized using SPE confocal microscope (Leica). Pictures were analyzed using CellProfiler.

\section{Statistical analysis}

Results were statistically evaluated using either the one-way ANOVA or unpaired t-test using GraphPad PrismTM version 6.0 software (GraphPad Software Inc., San Diego, CA, USA). The results are displayed as the means and SEM. The value of $* \mathrm{p}<0.05$ was considered as significant, and $* * \mathrm{p}<0.01$ or $* * * \mathrm{p}<0.001$ as highly significant.

\section{RNA-seq and ChIP-seq reads quality}

Quality of RNA-seq reads was assessed with Fastqc v0.11.8, Fastq-screen (Wingett and Andrews, 2018) v0.13.0 and MultiQC (Ewels et al., 2016) v1.7.

\section{RNA-seq}

RNA quantification. Salmon (Patro et al., 2017) tool v0.14.1 was used to quantify mm10 
NCBI RNA reference sequences (O’Leary et al., 2016) (RefSeq Curated, last updated 201711-16) downloaded from UCSC Table Browser (Karolchik et al., 2004). Salmon was launched with the following parameters : $\quad$--numBootstraps 60 --libType A -validateMappings.

Differential gene expression analysis. Statistical analysis was performed using R v3.5.1. Transcript expression levels were aggregated in Gene expression levels using tximport Bioconductor package (Soneson et al., 2015) v1.13.16. Deseq2 (Love et al., 2014) v1.22.2 method was used to identify differentially expressed genes between groups with a p-value threshold of 0.05 .

Permutation test. To create the list of genes hosting an L1Md, BED files containing L1Md genomic localizations (reconstructed Repbase from (Walter et al., 2016)) were intersected with the refseq_curated database from UCSC. Permutation test $(n=10,000)$ between lists of genes hosting an L1Md and DEG in IR vs NIR, or the same number of random genes (randomly extracted from Refseq without DEG) was performed using R studio and considered significant if $p<0.01$.

Motif Enrichment Analysis was performed using BaMM! web interface (Siebert and Söding, 2016; Kiesel et al., 2018) and de novo motif discovery module. Query motif was matched to known motifs using the hocomoco mouse database

GSEA Analysis was performed using Hallmark Gene Sets V7. To plot graphs, - $\log 10$ pValue is set to 4 when $\mathrm{p}<0.0001$.

\section{ChIP-seq}

Alignment. Human sequences were found in Mouse ChIP-seq reads. The contamination was removed with Xenome (Conway et al., 2012) v1.0.0. After contamination removal, ChIP-seq 
sequence reads were mapped to the Mouse genome build mm10 by using Burrows-Wheeler Aligner MEM algorithm ( $\mathrm{Li}$ and Durbin, 2009) (BWA v0.7.17). The read group ID was attached to every read in the resulting alignment file (bam file) with the -R parameter, and shorter split hits were marked as secondary with -M. Samtools (Li et al., 2009) fixmate v1.9 was used to check mate-pair information between mates and fixed if needed on a name sorted bam file. The duplicate reads were tagged by samtools markduplicates using a position sorted bam file. Secondary alignments and unmapped reads have been filtered out and only properly paired reads have been kept. Two types of downstream analysis have been performed, with multimapped reads (mapping quality score $>=0$ ) and one with uniquely mapped reads (mapping quality score $>=1$ ). Cross-correlation scores (NSC and RSC) have been calculated by phantompeakqualtools package (Kharchenko et al., 2008; Landt et al., 2012) v1.2. DeepTools (Ramírez et al., 2016) bamCoverage v3.3.0 has been used to generate normalized bigwig files with the following parameters : --binSize 1 --normalizeUsing BPM -extendReads -ignoreDuplicates. Then deepTools bigwigCompare was used to substract input signal from chip signal.

Peak calling. Areas in the genome enriched with aligned reads (also called peaks) were identified with MACS2 (Zhang et al., 2008) callpeak v2.1.2 with the following parameters : -f BAMPE -g mm10 -q 0.05 --broad --broad-cutoff 0.05 for H3K9me3 broad mark.

IDR (Irreproducible Discovery Rate) analysis. To measure the reproducibility between replicate experiments, we used the IDR method (Li et al., 2011) v2.0.4.2 with the following parameters : --rank q.value --random-seed 12345 --plot. Peaks with a global IDR score $<0.05$ were selected and used for downstream analysis.

Peak annotation. Annotatr 1.8.0 (R3.5.1) was used for peak annotation. 
H3K9me3 quantification and differential binding. To quantify H3K9me3 concentration at TE or promoters $(-2 \mathrm{~kb} ;+1 \mathrm{~kb}$ TSS), the Bioconductor package Diffbind (Ross-Innes et al., 2012) v2.10 was used in R v3.5.1. Paired-end mode was activated for read counting step with SummarizeOverlaps method. The default mapping quality threshold (mapQCth) was modified in 0 for multimapping analysis or 1 for unique mapping analysis. DBA_DESEQ2_BLOCK method was used to consider unwanted variable during normalization. Normalized H3K9me3 concentration at all TE loci from a same family/subfamily was summed to get a total H3K9me3 concentration per TE family. The age of a TE was calculated as in (Sookdeo et al., 2013): divergences were converted to time assuming a neutral rodent genomic substitution rate of $1.1 \% / \mathrm{MY}$.

Differential binding at peaks was identified with a p-value threshold of 0.05 .

Heatmaps. To plot heatmaps of $\mathrm{H} 3 \mathrm{~K} 9 \mathrm{me} 3$ enrichment at peaks, deeptools package v3.2.0 was used in $\mathrm{R}$ v3.5.1. The peaks (IDR $<0.05)$ files obtained for NIR and IR conditions were first fused using bedops. A matrix was then built using ComputeMatrix tool in the scaleregions mode between the generated fused bed file and the corresponding normalized bigwig files after input substraction. A body length of $2.5 \mathrm{~kb}$ (mean size of the peaks) was selected, as well as a $4 \mathrm{~kb}$ distance upstream and downstream of the start and the end of the peak. A filesorted region was generated and used to assess the presence or the absence of TE after computing a matrix with the TE genome coverage bigwig.

TE genome coverage. To generate TE genome coverage, bedtools package v2.27.1 was used. -bga option on the genomeCoverageBed tool was used. The bedGraph generated were then converted to bigwig files using the bedGraphToBigWig tool.

Accession numbers. ChIP-seq and RNA-seq raw data deposition on Annotare (ArrayExpress) is ongoing. 


\section{FIGURES LEGENDS}

Figure 1. H3K9me3 is mainly enriched at recent L1Md subfamilies in HSCs. (A) Experimental and bioinformatic analysis design for H3K9me3 ChIP-seq. Mice were subjected to 2 Gy total body irradiation (IR) or left untreated (NIR). Analysis was done on both Unique and Multiple mapping reads (U- and M-MRA respectively). (B) number of peaks with IDR score $<0.05$ in U- and M-MRA and in NIR and IR conditions. (C) Repartition of confident peak annotations in each genomic feature using annotateR in NIR and IR conditions and for U- and M-MRA. (D) repartition of $\mathrm{H} 3 \mathrm{~K} 9 \mathrm{me} 3$ concentration over the different $\mathrm{TE}$ families for M-MRA in NIR and IR. (E) Correlation plot representing H3K9me3 concentration quantified at all LINE elements in M-MRA $v s$ their age in million years (My). R, Pearson correlation coefficient ; p, pvalue.

Figure 2 : Irradiation induces a loss of H3K9me3 at L1Md subfamilies. (A) MA-plots showing non-significant (blue dots) and significant ( $p<0.05$ - pink dots) differential H3K9me3 enrichment at confident peaks between NIR and IR conditions analyzed both in U and MMRA. The number of peaks showing a significant decreased (down) or increased (up) in H3K9me3 enrichment upon IR is indicated in the plot. (B) Heatmap of H3K9me3 enrichment at M-MRA confident peaks in NIR and IR conditions. Each row represents one-scaled H3K9me3 peak with $+/-4 \mathrm{~kb}$ flanking regions. Genomic coverage of H3K9me3 peaks at different TE families and subfamilies are also represented. Blue, TE is present; yellow, TE is absent in the peak. (C) Plot profile of H3K9me3 enrichment along the L1Md sequences $(>5 \mathrm{~kb})+/-1 \mathrm{~kb}$ flanking regions in IR (green ) vs NIR (blue) conditions. (D) H3K9me3 enrichment at L1Md promoters analyzed by ChIP-qPCR normalized to H3K9me3 enrichment at repetitive 5S rRNA. $n=3$ independent experiments. Each dot represents pools of 3 (NIR) or 
4 (IR) mice. Results are expressed as fold change from the mean value of the NIR condition and represented as means $+/-\operatorname{SEM}(* p<0,05 ; * * p<0.01$ t-test $)$.

Figure 3. Irradiation induces a strong deregulation of the HSC transcriptome. (A) Volcano plot of the DEG between IR and NIR conditions. The horizontal axis represents the $\log 2$ fold change (FC) and the vertical axis the $-\log 10$ (pValue). Significantly $(p<0.05)$ upregulated (red) and downregulated (green) genes are shown. (B) Repartition of total $(p<0.05)$ DEG (grey); downregulated (green) or upregulated (red) genes in IR vs NIR according to their fold change. (C-H) GSEA analysis using Gene Sets. Significant $(p<0.05)$ gene sets gained $(\mathbf{C})$ or lost $(\mathbf{D})$ in the IR condition compared to NIR. $\log 10$ (pValue) is set to 4 when $p<0.001$. Enrichment plots for TNF- $\alpha \_$signaling_via_NFKB hallmark (E), HSC prosurvival TNF- $\alpha(\mathbf{F})$ and GMP TNF- $\alpha(\mathbf{G})$ gene signatures. (H) Enrichment plots for LTHSC; Low-output and dormant $v s$ activated HSC signatures.

Figure 4. Gene repression upon IR is associated with intronic L1Md. (A) Correlation plot representing the $\log 2$ (Fold change) in $\mathrm{H} 3 \mathrm{~K} 9 \mathrm{me} 3$ concentration at gene promoters $v s$ $\log 2$ (Fold change) in gene expression. (B-F) Permutation test comparing of the number of genes found in common between the list of DEG or 10000 lists of random genes and the list of genes hosting one or several L1Md. Blue curve: Distribution of the number of genes found in common between random genes and genes hosting an L1. Black vertical line: number of genes found in common between DEG and genes hosting an L1Md (C) or an Lx5 (D); or between genes downregulated (E) or upregulated (F) upon IR and hosting an L1Md. Significance bars $(p<0.01)$ are shown in red. (H-I) Heatmaps of the expression of genes from two LT-HSC signatures that are significantly up- (red) or down- (blue) regulated in IR vs NIR. Green stars indicate the presence of an intronic L1Md in the downregulated gene. 
Figure 5. Gene repression upon IR is associated with loss of H3K9me3 at intronic L1Md loci harboring NF-KB binding sites. (A, B) Experimental design and mRNA expression assessed by RT-qPCR in HSCs one month post TBI. Ct values were normalized to RPL32 and HPRT. Results are expressed as fold change from the mean value of the NIR condition. Each dot represents a pool of 3 (NIR) or 4 (IR) mice. Means +/- SEM from 3 to 4 independent experiments. (C, D) Experimental design and H3K9me3 ChIP-qPCR enrichment 1 month post TBI. The primer positioning at the intronic L1Md allowing the amplification of a unique and specific product is shown (C). Each dot represents a pool of 3 (NIR) or 4 (IR) mice from 2-4 independent experiments. Results are means +/- SEM of the percentage of input normalized to the NIR condition. ${ }^{* * *} \mathrm{p}<0.001 ;{ }^{* *} \mathrm{p}<0.01 ; * \mathrm{p}<0.05$ t-test. (E-F) De novo motif discovery analysis performed with the BaMMmotif tool on L1Md sequences located in introns of downregulated genes $v s$ non-deregulated genes $(\mathbf{E})$ or the genes participating $v s$ not participating to the loss of the LT-HSC signature (F). Enriched motifs were matched to known motifs using the Hocomoco mouse database.

Figure 6. TNF- $\alpha$ treatment prevents loss of $\mathrm{H3H} 9 \mathrm{me} 3$ at intronic L1Md, HSC gene repression and HSC loss of function. (A-C) NFKB1 expression in HSCs one month after TBI. (B) mRNA expression measured by RT-qPCR. Ct values were normalized to mean of RPL32 and HPRT. Results are expressed as fold change from the mean value of the NIR condition. Means +/- SEM. Each dot represents a pool of 3 (NIR) or 4 (IR) mice from 3 independent experiments. ${ }^{*} \mathrm{p}<0.05$ t-test. (C) Representative images and quantification of Nfkb1 protein mean IF intensity. Bars, $5 \mu \mathrm{M}$. Each dot represents a cell. Results are expressed as fold change from the mean value of the NIR condition from 2 independent experiments and represented as means $+/-$ SEM.*** $\mathrm{p}<0.001 \mathrm{t}$-test. (D-G) Experimental design analyzing the 
effects of IR and TNF- $\alpha$ in vitro. (E) Representative images and quantification of Nfkb1 staining. Bars, $5 \mu \mathrm{M}$. Each dot represents a cell. Results are represented as mean $+/-$ SEM of Nfkb1 IF intensity. One-way ANOVA with Tukey's multiple comparison test. (F) Gene expression evaluated by RT-qPCR. Means +/- SEM from 2 independent experiments. Oneway ANOVA with Tukey's multiple comparison test. (G) H3K9me3 enrichment at intronic L1Md evaluated by ChIP-qPCR. Results are expressed as in legend to Figure 5B. Means +/SEM from 2-3 independent experiments. One-way ANOVA with Bonferroni's multiple comparison test.

(H) Experimental design for reconstitution experiments using HSC sorted from mice one month after TBI and previously treated with TNF-a (IR+TNF) or not (IR), or left untreated (NIR); BMMC, bone marrow mononuclear cells. (I-J) percentage of GFP-negative donor contribution in blood in mice transplanted with NIR, IR or IR+TNF cells at 7 (I) and 14

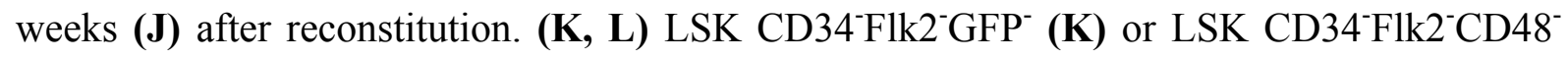
(L) GFP-negative donor HSC contribution in the BM 14 weeks after reconstitution. One-way ANOVA Tukey's multiple comparison test. ${ }^{*} \mathrm{p}<0.05 ;{ }^{*} \mathrm{p}<0.01 ; * * * \mathrm{p}<0.001 ; * * * * \mathrm{p}<0.0001$. (F) Model. At basal, the NF-KB pathway, possibly through its repressor NFKB1 (p50/p50 homodimers), is involved in the recruitment of H3K9 methylases (HMT) at intronic L1Md enriched in NFKB binding sites motifs, and apposition of the repressive histone mark H3K9me3. H3K9me3 "islands" into the body of transcribed genes may help the processing of RNAPolymerase II (RNAPolII) and transcript stability. Upon Irradiation, loss of the TNF-aNF-KB pathway leads to a loss of $\mathrm{H} 3 \mathrm{~K} 9 \mathrm{me} 3$ at the intronic $\mathrm{L} 1 \mathrm{Md}$, gene repression and transcript stability. Alternatively, loss of $\mathrm{H} 3 \mathrm{~K} 9 \mathrm{me} 3$ at intronic L1Md may lead to their transcription and gene repression through transcriptional interference. 


\section{Acknowledgments}

We thank animal facility, the Genomic and the Imaging and Cytometry Platforms of Gustave Roussy for RNA sequencing and cell sorting and confocal analysis, respectively; S. Gregoricchio and Drs. C. Lobry and C. Guillouf (INSERM U1170, Gustave Roussy) for their advices for ChIP-seq analysis, D. Dubray for Statistical analysis, and Drs M. Goodhardt and D. Garrick (INSERM UMRS-1126, Paris) for helpful discussions. We also thank A. Teissandier and D. Bourc'his for providing us the reconstructed repeatMasker database. This work was supported by INSERM and grants from Ligue National Contre le Cancer (LNCC, Equipe labellisée EL2020) and Institut National du Cancer (PLBIO N²020-095) to F. P., ARC Foundation (N 20161204988), GEGLUC Paris-Ile de France (2017) LNNC (20182019) and Agence National de la Recherche (ANRJCJC20-CE14-0018-01) to E.E.M. Y.P. and D.H. are recipients of fellowship from the Ministère de l'Enseignement Supérieur de la Recherche et de l'Innovation. A.S. is recipient of a fellowship from LNCC. F.H. is supported by INCA (PLBIO N²020-095 to F.P.).

The authors declare no conflict of interest.

\section{Author contributions}

Y.P., and E.E.M. performed the RNA-seq, ChIP-seq, ChIP-qPCR, experiments and analyzed the results. M.K.D., A.M.C and E.E.M. performed bioinformatic analyses. D.H., F.H., and A.S performed IF, qPCR and reconstitution experiments and analyzed the results. E.E.M. and F.P. designed and supervised the study, analyzed the results and wrote the manuscript 


\section{REFERENCES}

Adelman, E.R., Huang, H.-T., Roisman, A., Olsson, A., Colaprico, A., Qin, T., Lindsley, R.C., Bejar, R., Salomonis, N., Grimes, H.L., and Figueroa,M.E. (2019). Aging Human Hematopoietic Stem Cells Manifest Profound Epigenetic Reprogramming of Enhancers That May Predispose to Leukemia. Cancer Discov. 9, 1080-1101.

Adoue, V., Binet, B., Malbec, A., Fourquet, J., Romagnoli, P., van Meerwijk, J.P.M., Amigorena, S., and Joffre, O.P. (2019). The Histone Methyltransferase SETDB1 Controls T Helper Cell Lineage Integrity by Repressing Article The Histone Methyltransferase SETDB1 Controls T Helper Cell Lineage Integrity by Repressing Endogenous Retroviruses. Immunity. $50,629-644$.

Aguilera, A., and Gaillard, H. (2014). Transcription and Recombination: When RNA Meets DNA. Cold Spring Harb. Perspect. Biol. 6, a106543.

Antonaki, A., Demetriades, C., Polyzos, A., Banos, A., Vatsellas, G., Lavigne, M.D., Apostolou, E., Mantouvalou, E., Papadopoulou, D., Mosialos, G., and Thanos, D. (2011). Genomic analysis reveals a novel nuclear factor- $\mathrm{BB}(\mathrm{NF}-\mathrm{\kappa B})$-binding site in Alu-repetitive elements. J. Biol. Chem. 286, 38768-38782.

Aporntewan, C., Phokaew, C., Piriyapongsa, J., Ngamphiw, C., Ittiwut, C., Tongsima, S., and Mutirangura, A. (2011). Hypomethylation of intragenic LINE-1 represses transcription in cancer cells through AGO2. PLoS One 6.

Attig, J., Agostini, F., Gooding, C., Chakrabarti, A.M., Singh, A., Haberman, N., Zagalak, J.A., Emmett, W., Smith, C.W.J., Luscombe, N.M., and Ule, J. (2018). Heteromeric RNP assembly at LINEs controls lineage-specific RNA processing. Cell 174, 1067-1081.

Bailly-Bechet, M., Haudry, A., and Lerat, E. (2014). "One code to find them all": A perl tool to conveniently parse RepeatMasker output files. Mob. DNA 5, 13.

Barau, J., Teissandier, A., Zamudio, N., Roy, S., Nalesso, V., Hérault, Y., Guillou, F., and Bourc'his, D. (2016). The novel DNA methyltransferase DNMT3C protects male germ cells from transposon activity. Science 354, 909-912.

Barbieri, D., Elvira-Matelot, E., Pelinski, Y., Genève, L., de Laval, B., Yogarajah, G., Pecquet, C., Constantinescu, S.N., and Porteu, F. (2018). Thrombopoietin protects hematopoietic stem cells from retrotransposon-mediated damage by promoting an antiviral response. J. Exp. Med. 215, 1463-1480.

Bulut-Karslioglu, A., DeLaRosa-Velazquez, I.A., Ramirez, F., Barenboim, M., OnishiSeebacher, M., Arand, J., Galan, C., Winter, G.E., Engist, B., Gerle, B., et al. (2014). Suv39hDependent H3K9me3 Marks Intact Retrotransposons and Silences LINE Elements in Mouse Embryonic Stem Cells. Mol. Cell 55, 277-290.

Cabezas-Wallscheid, N., Buettner, F., Sommerkamp, P., Klimmeck, D., Ladel, L., Thalheimer, F.B., Pastor-Flores, D., Roma, L.P., Renders, S., Zeisberger, P., et al. (2017). Vitamin A-Retinoic Acid Signaling Regulates Hematopoietic Stem Cell Dormancy. Cell 169, 807-823.e19. 
Cartwright, T., Perkins, N.D., and L Wilson, C. (2016). NFKB1: a suppressor of inflammation, ageing and cancer. FEBS J. 283, 1812-1822.

Cartwright, T.N., Worrell, J.C., Marchetti, L., Dowling, C.M., Knox, A., Kiely, P., Mann, J., Mann, D.A., and Wilson, C.L. (2018). HDAC1 interacts with the p50 NF-kB subunit via its nuclear localization sequence to constrain inflammatory gene expression. Biochim. Biophys. Acta - Gene Regul. Mech. 1861, 962-970.

Chambers, S.M., Shaw, C.A., Gatza, C., Fisk, C.J., Donehower, L.A., and Goodell, M.A. (2007). Aging hematopoietic stem cells decline in function and exhibit epigenetic dysregulation. PLoS Biol. 5, 1750-1762.

Chuong, E.B., Elde, N.C., and Feschotte, C. (2016). Regulatory evolution of innate immunity through co-option of endogenous retroviruses. Science 351, 1083-1087.

Chuong, E.B., Elde, N.C., and Feschotte, C. (2017). Regulatory activities of transposable elements: from conflicts to benefits. Nat Rev Genet. 18, 71-86.

Conway, T., Wazny, J., Bromage, A., Tymms, M., Sooraj, D., Williams, E.D., and BeresfordSmith, B. (2012). Xenome--a tool for classifying reads from xenograft samples. Bioinformatics 28, i172-178.

Criscione, S.W., Teo, Y.V., and Neretti, N. (2016). The Chromatin Landscape of Cellular Senescence. Trends Genet. 32, 751-761.

Cuellar, L., Herzner, A.M., Zhang, X., Goyal, Y., Watanabe, C., Friedman, B.A., Janakiraman, V., Durinck, S., Stinson, J., Arnott, D., et al. (2017). Silencing of retrotransposons by SET DB1 inhibits the interferon response in acute myeloid leukemia. J. Cell Biol. 216, 3535-3549.

De La Mata, M., Alonso, C.R., Kadener, S., Fededa, J.P., Blaustein, M., Pelisch, F., Cramer, P., Bentley, D., and Kornblihtt, A.R. (2003). A slow RNA polymerase II affects alternative splicing in vivo. Mol. Cell 12, 525-532.

De Laval, B., Pawlikowska, P., Petit-Cocault, L., Bilhou-Nabera, C., Aubin-Houzelstein, G., Souyri, M., Pouzoulet, F., Gaudry, M., and Porteu, F. (2013). Thrombopoietin-increased DNA-PK-dependent DNA repair limits hematopoietic stem and progenitor cell mutagenesis in response to dna damage. Cell Stem Cell 12, 37-48.

De Laval, B., Pawlikowska, P., Barbieri, D., Besnard-Guerin, C., Cico, A., Kumar, R., Gaudry, M., Baud, V., and Porteu, F. (2014). Thrombopoietin promotes NHEJ DNA repair in hematopoietic stem cells through specific activation of Erk and NF-kB pathways and their target, IEX-1. Blood 123, 509-519.

Deniz, Ö., Ahmed, M., Todd, C.D., Rio-Machin, A., Dawson, M.A., and Branco, M.R. (2020). Endogenous retroviruses are a source of enhancers with oncogenic potential in acute myeloid leukaemia. Nat. Commun. 11, 3506-3520.

Djeghloul, D., Kuranda, K., Kuzniak, I., Barbieri, D., Naguibneva, I., Choisy, C., Bories, J.- 
C., Dosquet, C., Pla, M., Vanneaux, V., et al. (2016). Age-Associated Decrease of the Histone Methyltransferase SUV39H1 in HSC Perturbs Heterochromatin and B Lymphoid Differentiation. Stem Cell Reports 6, 970-984.

Ea, C.K., Hao, S.L., Yeo, K.S., and Baltimore, D. (2012). EHMT1 protein binds to nuclear factor- $\mathrm{KB}$ p50 and represses gene expression. J. Biol. Chem. 287, 31207-31217.

Elsharkawy, A.M., Oakley, F., Lin, F., Packham, G., Mann, D.A., and Mann, J. (2010). The NF- $\kappa$ B p50:p50:HDAC-1 repressor complex orchestrates transcriptional inhibition of multiple pro-inflammatory genes. J. Hepatol. 53, 519-527.

Ewels, P., Magnusson, M., Lundin, S., and Käller, M. (2016). MultiQC: summarize analysis results for multiple tools and samples in a single report. Bioinformatics 32, 3047-3048.

Fang, J., Muto, T., Kleppe, M., Bolanos, L.C., Hueneman, K.M., Walker, C.S., Sampson, L., Wellendorf, A.M., Chetal, K., Choi, K., et al. (2018). TRAF6 Mediates Basal Activation of

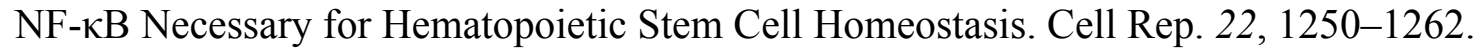

Faulkner, G.J., Kimura, Y., Daub, C.O., Wani, S., Plessy, C., Irvine, K.M., Schroder, K., Cloonan, N., Steptoe, A.L., Lassmann, T., et al. (2009). The regulated retrotransposon transcriptome of mammalian cells. Nat. Genet. 41, 563-571.

Fleenor, C.J., Rozhok ,A.I., Zaberezhnyy, V., Mathew, D., Kim, J., Tan, A., Bernstein, I.D., DeGregori, J. (2015). Contrasting roles for $\mathrm{C} / \mathrm{EBP} \alpha$ and Notch in irradiation-induced multipotent hematopoietic progenitor cell defects. Stem Cells 33, 1345-1358.

Gazquez-Gutierrez, A., Witteveldt, J., R. Heras, S., and Macias, S. (2021). Sensing of transposable elements by the antiviral innate immune system. Rna rna.078721.121.

Goodier, J.L., Pereira, G.C., Cheung, L.E., Rose, R.J., and Kazazian, H.H. (2015). The BroadSpectrum Antiviral Protein ZAP Restricts Human Retrotransposition. PLoS Genet. 11, 1-32.

Grundy, E.E., Diab, N., and Chiappinelli, K.B. (2021). Transposable element regulation and expression in cancer. FEBS J. Jan 20, doi: 10.1111/febs.15722.

Gu, Z., Liu, Y., Zhang, Y., Cao, H., Lyu, J., Wang, X., Wylie, A., Newkirk, S.J., Jones, A.E., Lee M., et al. 2021. Silencing of LINE-1 retrotransposons is a selective dependency of myeloid leukemia. Nat. Genet. 53, 672-682

Han, J.S., Szak, S.T., and Boeke, J.D. (2004). Transcriptional disruption by the L1 retrotransposon and implications for mammalian transcriptomes. Nature 429, 268-274.

Hermant, C., and Torres-Padilla, M.E. (2021). TFs for TEs: The transcription factor repertoire of mammalian transposable elements. Genes Dev. 35, 22-39.

Hu, M., Lu, Y., Zeng, H., Zhang, Z., Chen, S., Qi, Y., Xu, Y., Chen, F., Tang, Y., Chen, M., et al; (2020). MicroRNA-21 maintains hematopoietic stem cell homeostasis through sustaining the NF- $\mathrm{KB}$ signaling pathway in mice. Haematologica 105, 412-423.

Jachowicz, J.W., Bing, X., Pontabry, J., Bošković, A., Rando, O.J., and Torres-Padilla, M.E. 
(2017). LINE-1 activation after fertilization regulates global chromatin accessibility in the early mouse embryo. Nat. Genet. 49, 1502-1510.

Jang, H.S., Shah, N.M., Du, A.Y., Dailey, Z.Z., Pehrsson, E.C., Godoy, P.M., Zhang, D., Li, D., Xing, X., Kim, S., et al. (2019). Transposable elements drive widespread expression of oncogenes in human cancers. Nat. Genet. 51, 611-617.

Jones, P.A. (2012). Functions of DNA methylation: Islands, start sites, gene bodies and beyond. Nat. Rev. Genet. 13, 484-492.

Kaer, K., Branovets, J., Hallikma, A., Nigumann, P., and Speek, M. (2011). Intronic 11 retrotransposons and nested genes cause transcriptional interference by inducing intron retention, exonization and cryptic polyadenylation. PLoS One 6, e26099.

Karolchik, D., Hinrichs, A.S., Furey, T.S., Roskin, K.M., Sugnet, C.W., Haussler, D., and Kent, W.J. (2004). The UCSC Table Browser data retrieval tool. Nucleic Acids Res. 32, D493-496.

Kazachenka, A., Young, G.R., Attig, J., Kordella, C., Lamprianidou, E., Zoulia, E., Vrachiolias, G., Papoutselis, M., Bernard, E., Papaemmanuil, E., et al. (2019). Epigenetic therapy of myelodysplastic syndromes connects to cellular differentiation independently of endogenous retroelement derepression. Genome Med. 11,86

Keenan, C.R., Iannarella, N., Naselli, G., Bediaga, N.G., and Timothy, M. (2021). Extreme disruption of heterochromatin is required for accelerated haematopoietic aging. Blood., 135, 2049-2058.

Kelsey, M.M.G. (2021). Reconsidering LINE-1's role in cancer: Does LINE-1 function as a reporter detecting early cancer-Associated epigenetic signatures? Evol. Med. Public Heal. 9, 78-82.

Kharchenko, P. V., Tolstorukov, M.Y., and Park, P.J. (2008). Design and analysis of ChIPseq experiments for DNA-binding proteins. Nat. Biotechnol. 26, 1351-1359.

Kiesel, A., Roth, C., Ge, W., Wess, M., Meier, M., and Söding, J. (2018). The BaMM web server for de-novo motif discovery and regulatory sequence analysis. Nucleic Acids Res. 46, W215-W220.

Koide, S., Oshima, M., Takubo, K., Yamazaki, S., Nitta, E., Saraya, A., Aoyama, K., Kato, Y., Miyagi, S., Nakajima-Takagi, Y., et al. (2016). Setdb1 maintains hematopoietic stem and progenitor cells by restricting the ectopic activation of nonhematopoietic genes. Blood 128 , $638-649$.

Landt, S.G., Marinov, G.K., Kundaje, A., Kheradpour, P., Pauli, F., Batzoglou, S., Bernstein, B.E., Bickel, P., Brown, J.B., Cayting, P., et al. (2012). ChIP-seq guidelines and practices of the ENCODE and modENCODE consortia. Genome Res. 22, 1813-1831.

Lefkopoulos, S., Polyzou, A., Derecka, M., Bergo, V., Clapes, T., Cauchy, P., Jerez-Longres, C., Onishi-Seebacher, M., Yin, N., Martagon-Calderón, N.A. et al. (2020). Repetitive 
Elements Trigger RIG-I-like Receptor Signaling that Regulates the Emergence of Hematopoietic Stem and Progenitor Cells. Immunity 53, 934-951.e9.

Li, H., and Durbin, R. (2009). Fast and accurate short read alignment with Burrows-Wheeler transform. Bioinformatics 25, 1754-1760.

Li, H., Handsaker, B., Wysoker, A., Fennell, T., Ruan, J., Homer, N., Marth, G., Abecasis, G., and Durbin, R. (2009). The Sequence Alignment/Map format and SAMtools. Bioinformatics 25, 2078-2079.

Li, Q., Brown, J.B., Huang, H., and Bickel, P.J. (2011). Measuring reproducibility of highthroughput experiments. Ann. Appl. Stat. 5, 1752-1779.

Liu, N., Lee, C.H., Swigut, T., Grow, E., Gu, B., Bassik, M.C., and Wysocka, J. (2018). Selective silencing of euchromatic L1s revealed by genome-wide screens for L1 regulators. Nature 553, 228-232.

Love, M.I., Huber, W., and Anders, S. (2014). Moderated estimation of fold change and dispersion for RNA-seq data with DESeq2. Genome Biol. 15, 550.

Miousse, I.R., Kutanzi, K.R., and Koturbash, I. (2017a). Effects of ionizing radiation on DNA methylation: from experimental biology to clinical applications. Int. J. Radiat. Biol. 93, 457469.

Miousse, I.R., Chang, J., Shao, L., Pathak, R., Nzabarushimana, É., Kutanzi, K.R., Landes, R.D., Tackett, A.J., Hauer-Jensen, M., Zhou, D., and Koturbash, I. (2017b). Inter-Strain differences in LINE-1 DNA methylation in the mouse hematopoietic system in response to exposure to ionizing radiation. Int. J. Mol. Sci. 18, 1430-1446.

Moehrle, B.M., Nattamai, K., Brown, A., Florian, M.C., Ryan, M., Vogel, M., Bliederhaeuser, C., Soller, K., Prows, D.R., and Abdollahi, A. et al. (2015). Stem CellSpecific Mechanisms Ensure Genomic Fidelity within HSCs and upon Aging of HSCs. Cell Reports, 13, 2412-2424.

Moelling, K., and Broecker, F. (2019). Viruses and evolution - Viruses first? A personal perspective. Front. Microbiol. 10, 523.

Mohrin, M., Bourke, E., Alexander, D., Warr, M.R., Barry-Holson, K., Le Beau, M.M., Morrison, C.G., and Passegué, E. (2010). Hematopoietic stem cell quiescence promotes errorprone DNA repair and mutagenesis. Cell Stem Cell 7, 174-185.

Monaghan, L., Massett, M.E., Bunschoten, R.P., Hoose, A., Pirvan, P.A., Liskamp, R.M.J., Jørgensen, H.G., and Huang, X. (2019). The Emerging Role of H3K9me3 as a Potential Therapeutic Target in Acute Myeloid Leukemia. Front. Oncol. 9, 705.

Müller-Tidow, C., Klein, H.U., Hascher, A., Isken, F., Tickenbrock, L., Thoennissen, N., Agrawal-Singh, S., Tschanter, P., Disselhoff, C., Wang, Y., et al. (2010). Profiling of histone H3 lysine 9 trimethylation levels predicts transcription factor activity and survival in acute myeloid leukemia. Blood 116, 3564-3571. 
Ninova, M., Chen, Y.C.A., Godneeva, B., Rogers, A.K., Luo, Y., Fejes Tóth, K., and Aravin, A.A. (2020). Su(var)2-10 and the SUMO Pathway Link piRNA-Guided Target Recognition to Chromatin Silencing. Mol. Cell 77, 556-570.e6.

O’Leary, N.A., Wright, M.W., Brister, J.R., Ciufo, S., Haddad, D., McVeigh, R., Rajput, B., Robbertse, B., Smith-White, B., Ako-Adjei, D., et al. (2016). Reference sequence (RefSeq) database at NCBI: current status, taxonomic expansion, and functional annotation. Nucleic Acids Res. 44, D733-745.

Ocampo, A., Reddy, P., Martinez-Redondo, P., Platero-Luengo, A., Hatanaka, F., Hishida, T., Li, M., Lam, D., Kurita, M., Beyret, E. et al. (2016). In Vivo Amelioration of Age-Associated Hallmarks by Partial Reprogramming. Cell 167, 1719-1733.e12.

Patro, R., Duggal, G., Love, M.I., Irizarry, R.A., and Kingsford, C. (2017). Salmon provides fast and bias-aware quantification of transcript expression. Nat. Methods 14, 417-419.

Pezic, D., Manakov, S.A., Sachidanandam, R., and Aravin, A.A. (2014). piRNA pathway targets active LINE1 elements to establish the repressive H3K9me3 mark in germ cells. Genes Dev. 28, 1410-1428.

Pietras, E.M., Reynaud, D., Kang, Y.A., Carlin, D., Calero-Nieto, F.J., Leavitt, A.D., Stuart, J.A., Göttgens, B., and Passegué, E. (2015). Functionally Distinct Subsets of Lineage-Biased Multipotent Progenitors Control Blood Production in Normal and Regenerative Conditions. Cell Stem Cell 17, 35-46.

Ramírez, F., Ryan, D.P., Grüning, B., Bhardwaj, V., Kilpert, F., Richter, A.S., Heyne, S., Dündar, F., and Manke, T. (2016). deepTools2: a next generation web server for deepsequencing data analysis. Nucleic Acids Res. 44, W160-165.

Rodriguez-Fraticelli, A.E., Weinreb, C., Wang, S.W., Migueles, R.P., Jankovic, M., Usart, M., Klein, A.M., Lowell, S., and Camargo, F.D. (2020). Single-cell lineage tracing unveils a role for TCF15 in haematopoiesis. Nature 583, 585-589.

Ross-Innes, C.S., Stark, R., Teschendorff, A.E., Holmes, K.A., Ali, H.R., Dunning, M.J., Brown, G.D., Gojis, O., Ellis, I.O., Green, A.R.et al. (2012). Differential oestrogen receptor binding is associated with clinical outcome in breast cancer. Nature 481, 389-393.

Saint-andré, V., Batsché, E., Rachez, C., and Muchardt, C. (2011). Histone H3 lysine 9 trimethylation and HP1 G favor inclusion of alternative exons. Nat. Struct. Mol. Biol. 18, 337-344.

Sanjuan-Pla, A., Macaulay, I.C., Jensen, C.T., Woll, P.S., Luis, T.C., Mead, A., Moore, S., Carella, C., Matsuoka, S., Jones, T.B. et al. (2013). Platelet-biased stem cells reside at the apex of the haematopoietic stem-cell hierarchy. Nature 502, 232-236.

Schneider, W.M., Chevillotte, M.D., and Rice, C.M. (2014). Interferon-stimulated genes: A complex web of host defenses. Annu. Rev. Immunol. 32, 513-545.

Schaefer, B.C., Schaefer, M.L., Kappler, J.W., Marrack, P., and Kedl, R.M. (2001). Observation of antigen-dependent CD8+ T-cell/dendritic cell interactions in vivo. Cell. 
Immunol. 214, 110-122.

Schreiber, J., Jenner, R.G., Murray, H.L., Gerber, G.K., Gifford, D.K., and Young, R.A. (2006). Coordinated binding of NF- $\kappa B$ family members in the response of human cells to lipopolysaccharide. Proc. Natl. Acad. Sci. U. S. A. 103, 5899-5904.

Siebert, M., and Söding, J. (2016). Bayesian Markov models consistently outperform PWMs at predicting motifs in nucleotide sequences. Nucleic Acids Res. 44, 6055-6069.

Soneson, C., Love, M.I., and Robinson, M.D. (2015). Differential analyses for RNA-seq: transcript-level estimates improve gene-level inferences. F1000Research 4, 1521.

Sookdeo, A., Hepp, C.M., McClure, M.A., and Boissinot, S. (2013). Revisiting the evolution of mouse LINE-1 in the genomic era. Mob. DNA 4, 1-15.

Stein, S.J., and Baldwin, A.S. (2013). Deletion of the NF-kB subunit p65/RelA in the hematopoietic compartment leads to defects in hematopoietic stem cell function. Blood 121, 5015-5024.

Su, A.I., Cooke, M.P., Ching, K.A., Hakak, Y., Walker, J.R., Wiltshire, T., Orth, A.P., Vega, R.G., Sapinoso, L.M., Moqrich, A. et al.(2002). Large-scale analysis of the human and mouse transcriptomes. Proc. Natl. Acad. Sci. U. S. A. 99, 4465-4470.

Sun, D., Luo, M., Jeong, M., Rodriguez, B., Xia, Z., Hannah, R., Wang, H., Le, T., Faull, K.F., Chen, R. et al. (2014). Epigenomic profiling of young and aged HSCs reveals concerted changes during aging that reinforce self-renewal. Cell Stem Cell 14, 673-688.

Sun, X., Wang, X., Tang, Z., Grivainis, M., Kahler, D., Yun, C., Mita, P., Fenyö, D., and Boeke, J.D. (2018). Transcription factor profiling reveals molecular choreography and key regulators of human retrotransposon expression. Proc. Natl. Acad. Sci. U. S. A. 115, E5526E5535.

Sundaram, V., and Wang, T. (2018). Transposable Element Mediated Innovation in Gene Regulatory Landscapes of Cells: Re-Visiting the "Gene-Battery" Model. BioEssays 40, 10.1002/bies.201700155.

Takahashi, K., Wang, F., Kantarjian, P.H., Doss, D., Khanna, K., Thompson, E., Zhao, L., Patel, K., Neelapu, S., Gumbs, C. et al. (2018). Preleukaemic clonal haemopoiesis and risk of therapy-related myeloid neoplasms: a case-control study. Lancet Oncol. 18, 100-111.

Teissandier, A., Servant, N., Barillot, E., and Bourc'His, D. (2019). Tools and best practices for retrotransposon analysis using high-throughput sequencing data. Mob. DNA 10, 1-12.

Trizzino, M., Kapusta, A., and Brown, C.D. (2018). Transposable elements generate regulatory novelty in a tissue-specific fashion. BMC Genomics. 19, 1623-1633.

Tsurumi, A., and Li, W.X. (2012). Global heterochromatin loss: a unifying theory of aging? Epigenetics. 7, 680-688.

Vakoc, C.R., Mandat, S.A., Olenchock, B.A., and Blobel, G.A. (2005). Histone H3 Lysine 9 
Methylation and HP1gamma Are Associated with Transcription Elongation through Mammalian Chromatin. Mol. Cell. 19, 381-391.

Volkman, H.E., and Stetson, D.B. (2014). The enemy within: Endogenous retroelements and autoimmune disease. Nat. Immunol. 15, 415-422.

Wahlestedt, M., Erlandsson, E., Kristiansen, T., Lu, R., Brakebusch, C., Weissman, I.L., Yuan, J., Martin-Gonzalez, J., and Bryder, D. (2017). Clonal reversal of ageing-associated stem cell lineage bias via a pluripotent intermediate. Nat. Commun. 8, 14533.

Walter, M., Teissandier, A., Pérez-Palacios, R., and Bourc'his, D. (2016). An epigenetic switch ensures transposon repression upon dynamic loss of DNA methylation in embryonic stem cells. Elife 5, e11418.

Wang, K., Li, H., Xu, Y., Shao, Q., Yi, J., Wang, R., Cai, W., Hang, X., Zhang, C., Cai, H., and Qu, W. (2019). MFEprimer-3.0: Quality control for PCR primers. Nucleic Acids Res. 47, W610-W613.

Whitaker, J.W., Chen, Z., and Wang, W. (2015). Predicting the human epigenome from DNA motifs. Nat. Methods 12, 265-272.

White, R.R., and Vijg, J. (2016). Do DNA Double-Strand Breaks Drive Aging? Mol. Cell 63, 729-738.

Wingett, S.W., and Andrews, S. (2018). FastQ Screen: A tool for multi-genome mapping and quality control. F1000Research 7, 1338.

Yamashita, M., and Passegué, E. (2019). TNF- $\alpha$ Coordinates Hematopoietic Stem Cell Survival and Myeloid Regeneration. Cell Stem Cell 25, 357-372.e7.

Ye, M., Goudot, C., Hoyler, T., Lemoine, B., Amigorena, S., and Zueva, E. (2020). Specific subfamilies of transposable elements contribute to different domains of $\mathrm{T}$ lymphocyte enhancers. Proc. Natl. Acad. Sci. U. S. A. 117, 7905-7916.

Yu, Q., Carbone, C.J., Katlinskaya, Y. V., Zheng, H., Zheng, K., Luo, M., Wang, P.J., Greenberg, R.A., and Fuchs, S.Y. (2015). Type I interferon controls propagation of long interspersed element-1. J. Biol. Chem. 290, 10191-10199.

Zhang, Y., Liu, T., Meyer, C.A., Eeckhoute, J., Johnson, D.S., Bernstein, B.E., Nusbaum, C., Myers, R.M., Brown, M., Li, W., and Liu, X.S. (2008). Model-based analysis of ChIP-Seq (MACS). Genome Biol. 9, R137. 
A.

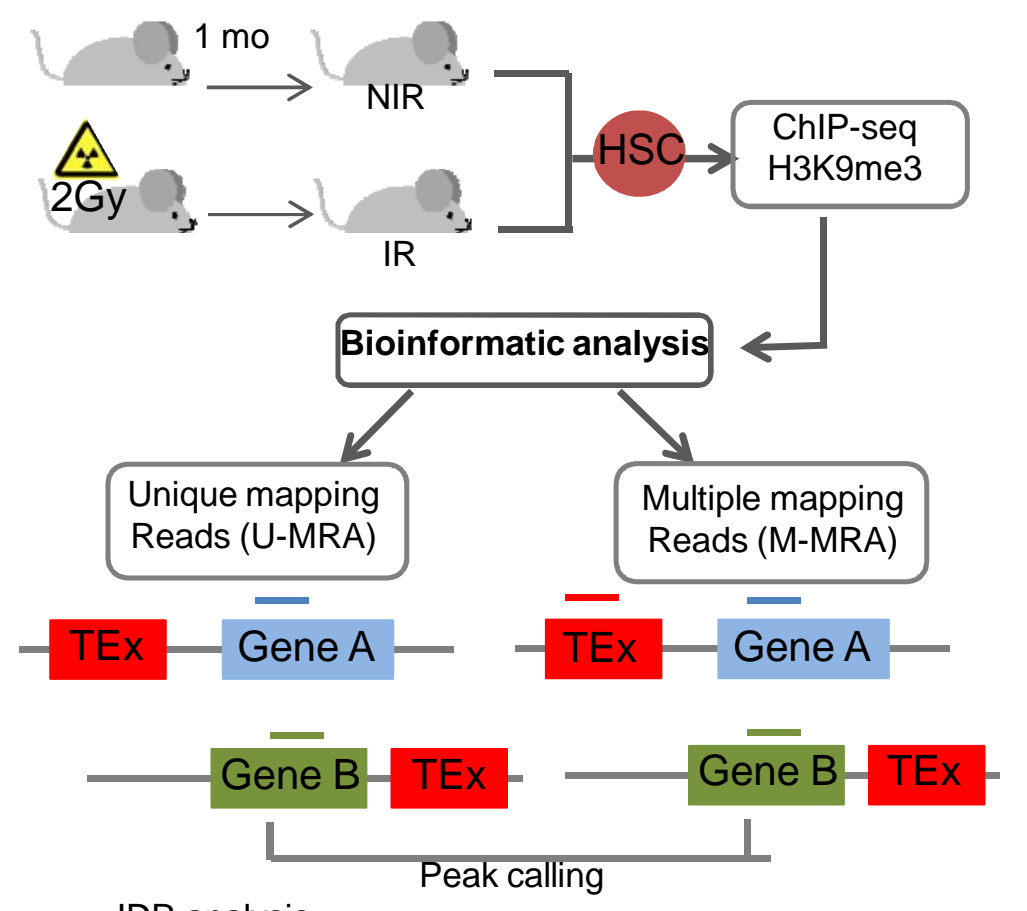

IDR analysis

Confident peaks annotation

Differential enrichment analysis on confident peaks

Differential enrichment analysis on RTE bed files

C.

NIR
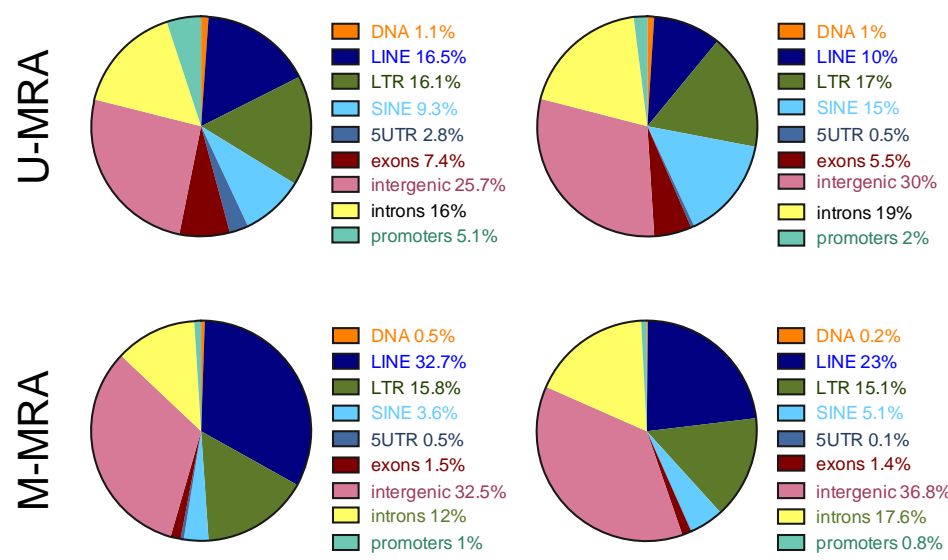

$\frac{\pi}{\sigma}$

อุ

\#



E.

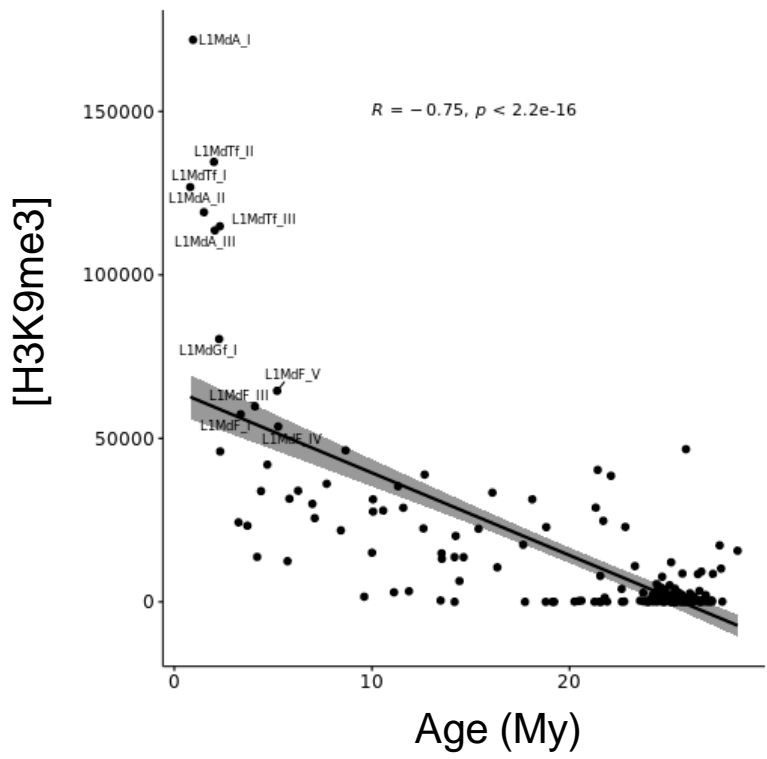

D.

Figure 1. H3K9me3 is mainly enriched at recent L1Md subfamilies in HSCs. (A) Experimental and bioinformatic analysis design for $\mathrm{H} 3 \mathrm{~K} 9 \mathrm{me} 3 \mathrm{ChIP}$-seq. Mice were subjected to $2 \mathrm{~Gy}$ total body irradiation (IR) or left untreated (NIR). Analysis was done on both Unique and Multiple mapping reads (U- and M-MRA respectively). (B) number of peaks with IDR score <0.05 in U- and M-MRA and in NIR and IR conditions. (C) Repartition of confident peak annotations in each genomic feature using annotateR in NIR and IR conditions and for U- and M-MRA. (D) repartition of H3K9me3 concentration over the different TE families for M-MRA in NIR and IR. (E) Correlation plot representing $\mathrm{H} 3 \mathrm{~K} 9 \mathrm{me} 3$ concentration quantified at all LINE elements in M-MRA vs their age in million years (My). R, Pearson correlation coefficient ; $p$, pvalue. 
Figure 2

A.
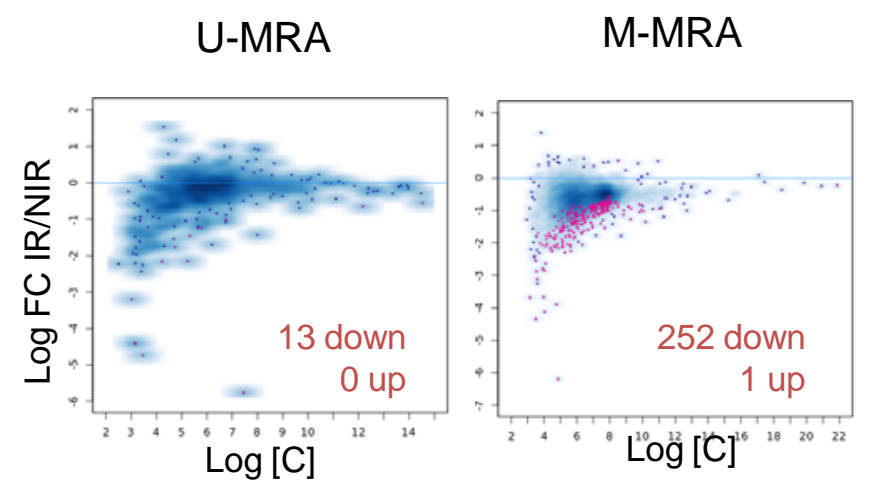

B.
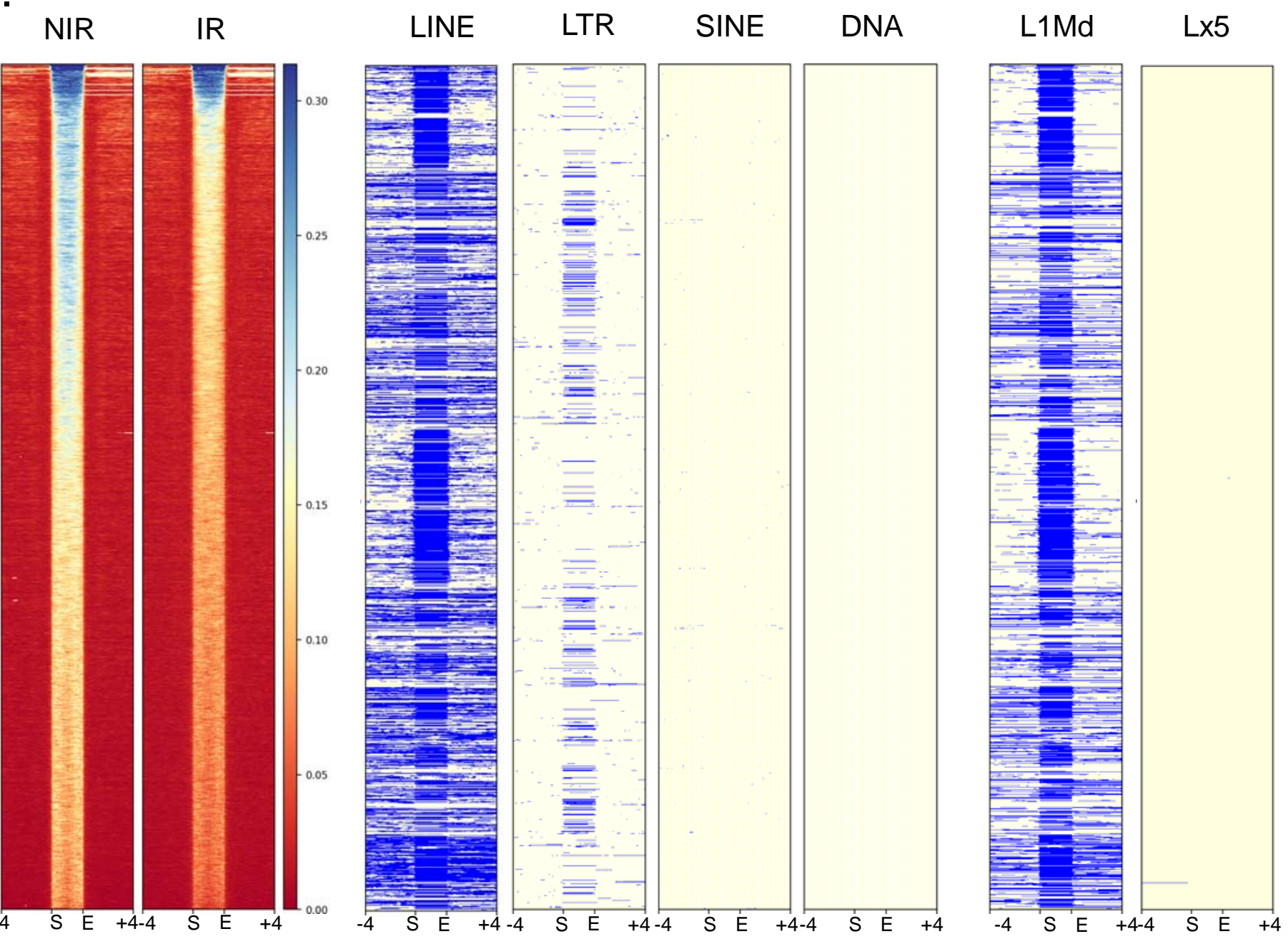

C.

D.
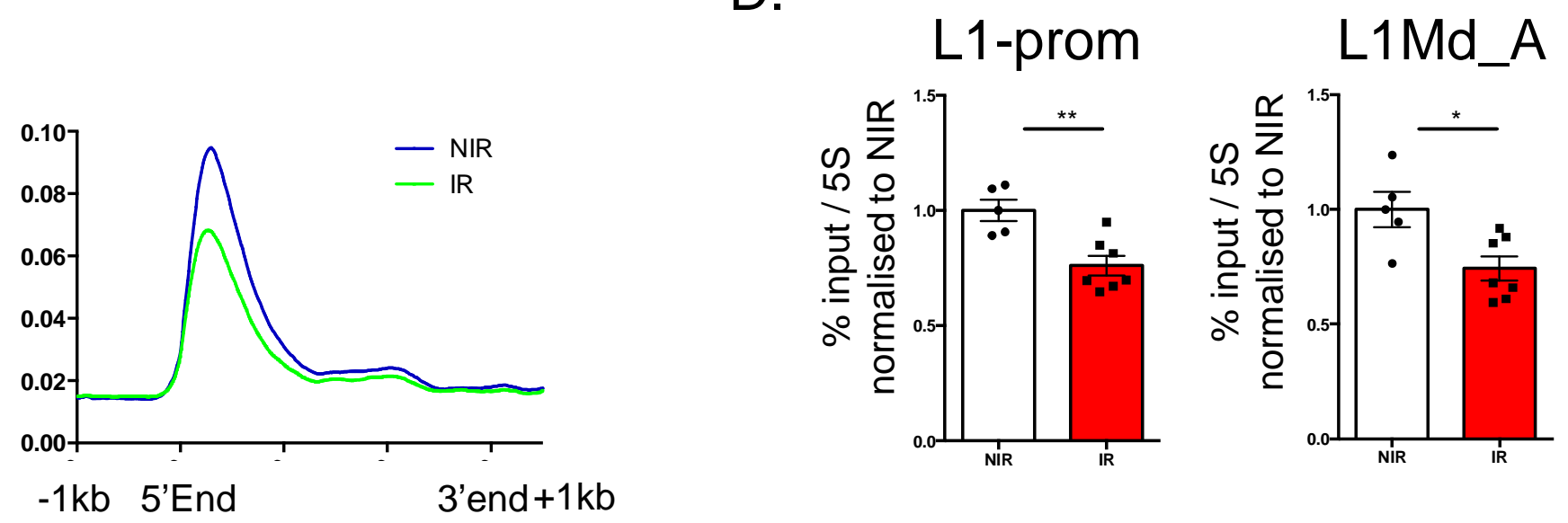
Figure 2 : Irradiation induces a loss of H3K9me3 at L1Md subfamilies. (A) MA-plots showing non-significant (blue dots) and significant ( $p<0.05$ - pink dots) differential H3K9me3 enrichment at confident peaks between NIR and IR conditions analyzed both in U and M-MRA. The number of peaks showing a significant decreased (down) or increased (up) in H3K9me3 enrichment upon IR is indicated in the plot. (B) Heatmap of H3K9me3 enrichment at M-MRA confident peaks in NIR and IR conditions. Each row represents one-scaled H3K9me3 peak with $+/-4 \mathrm{~kb}$ flanking regions. Genomic coverage of $\mathrm{H} 3 \mathrm{~K} 9 \mathrm{me} 3$ peaks at different TE families and subfamilies are also represented. Blue, TE is present; yellow, TE is absent in the peak. (C) Plot profile of H3K9me3 enrichment along the L1Md sequences $(>5 \mathrm{~kb})+/-1 \mathrm{~kb}$ flanking regions in IR (green ) vs NIR (blue) conditions. (D) H3K9me3 enrichment at L1Md promoters analyzed by ChIP-qPCR normalized to H3K9me3 enrichment at repetitive 5S rRNA. $n=3$ independent experiments. Each dot represents pools of 3 (NIR) or 4 (IR) mice. Results are expressed as fold change from the mean value of the NIR condition and represented as means $+/-\operatorname{SEM}(* \mathrm{p}<0,05 ; * * \mathrm{p}<0.01 \mathrm{t}$-test $)$. 


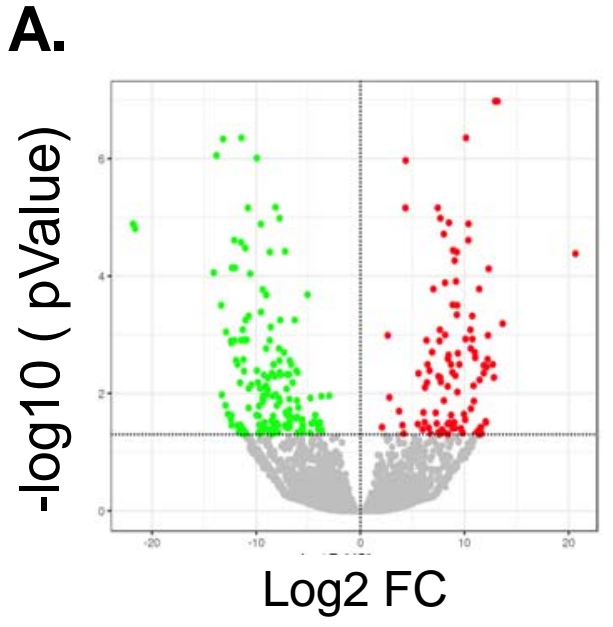

C.

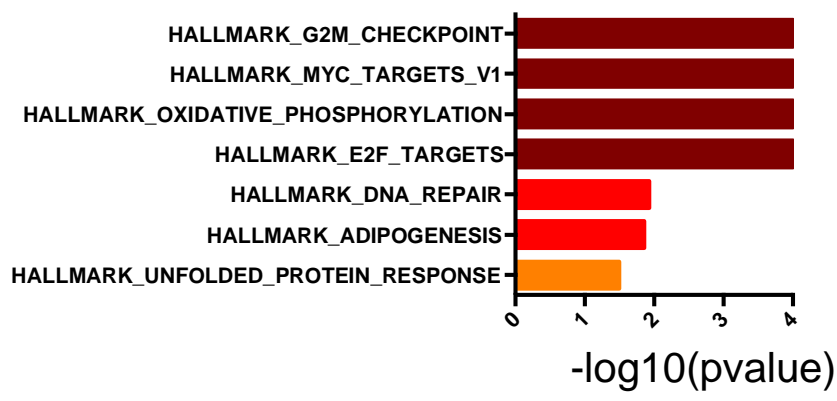

B.

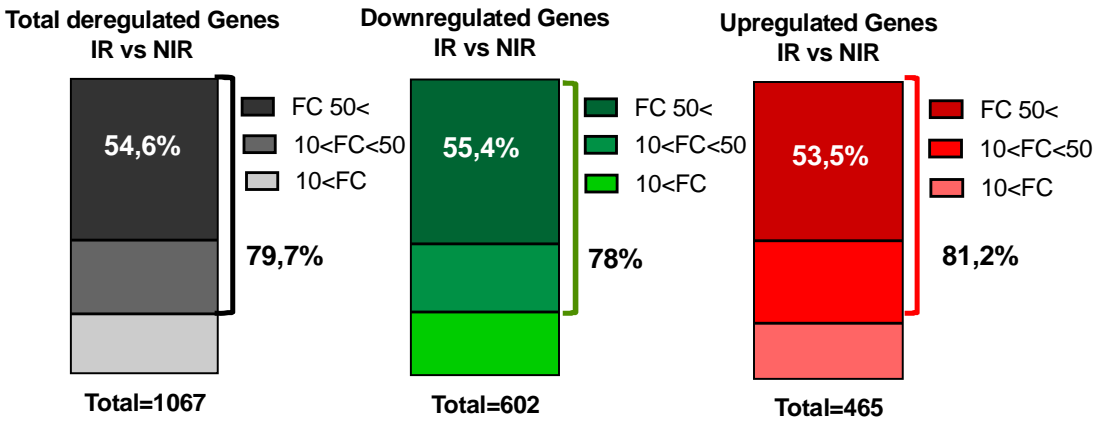

D.

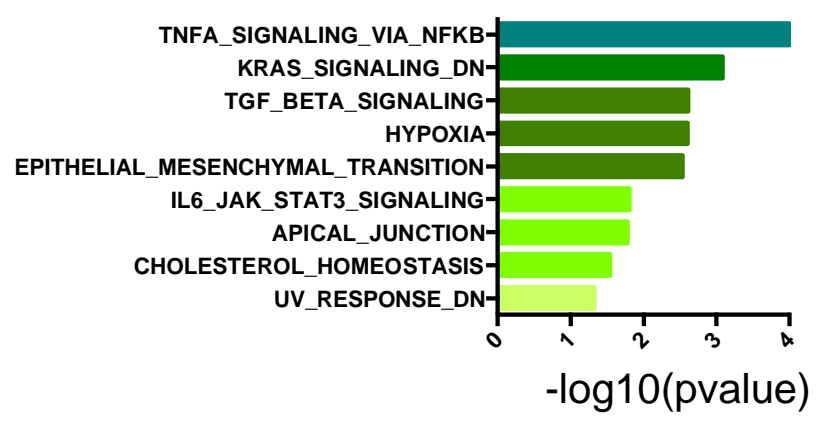

G.

F.

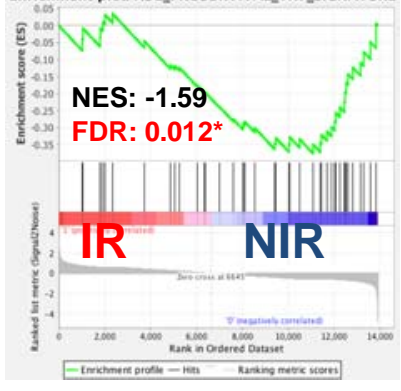

Enrichment plot: GMP_TNF_SIGNATURE

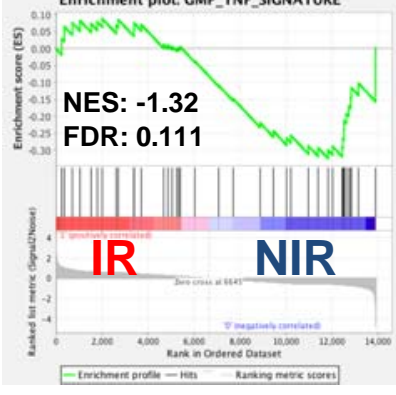

H.
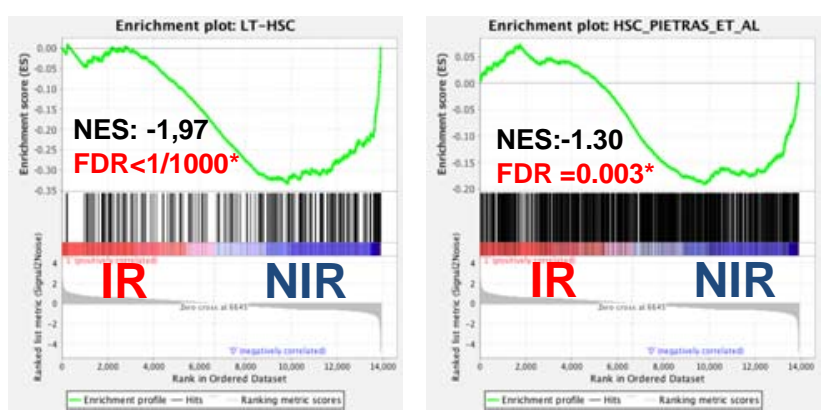

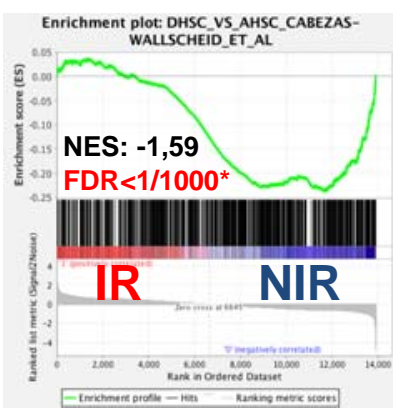


Figure 3. Irradiation induces a strong deregulation of the HSC transcriptome. (A) Volcano plot of the DEG between IR and NIR conditions. The horizontal axis represents the $\log 2$ fold change (FC) and the vertical axis the $-\log 10$ (pValue). Significantly $(p<0.05)$ upregulated (red) and downregulated (green) genes are shown. (B) Repartition of total $(p<0.05)$ DEG (grey); downregulated (green) or upregulated (red) genes in IR vs NIR according to their fold change. (CH) GSEA analysis using Gene Sets. Significant $(p<0.05)$ gene sets gained $(\mathbf{C})$ or lost $(\mathbf{D})$ in the IR condition compared to NIR. $\log 10$ (pValue) is set to 4 when $p<0.001$. Enrichment plots for TNFa_signaling_via_NFKB hallmark (E), HSC prosurvival TNF-a (F) and GMP TNF-a (G) gene signatures. (H) Enrichment plots for LT-HSC; Low-output and dormant vs activated HSC signatures. 

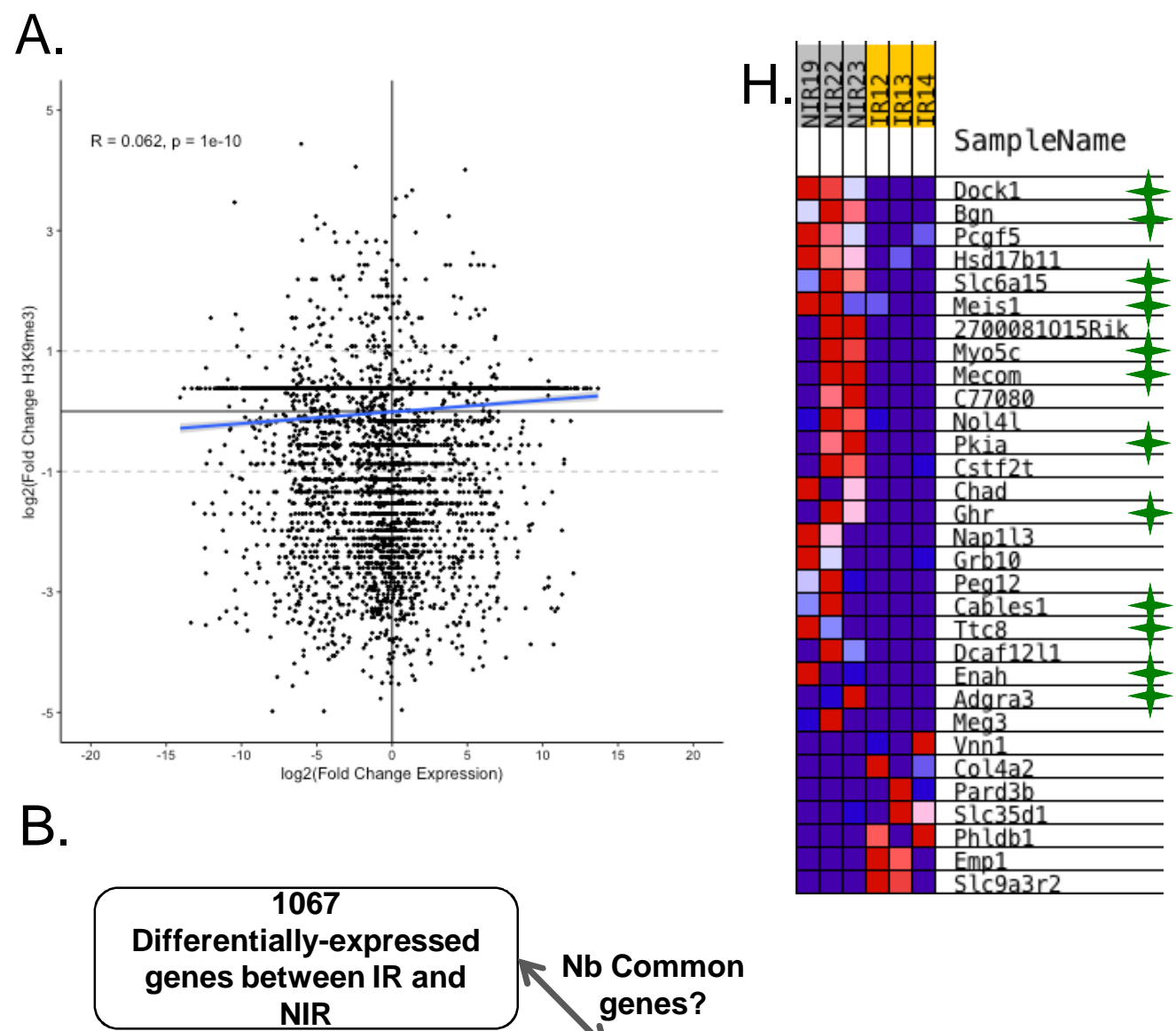

Nb Common
genes?

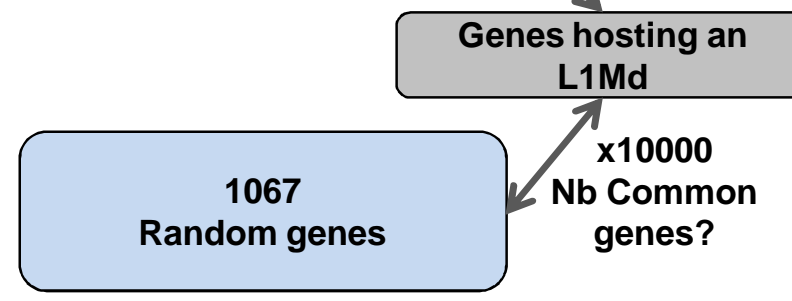

C.

L1Md

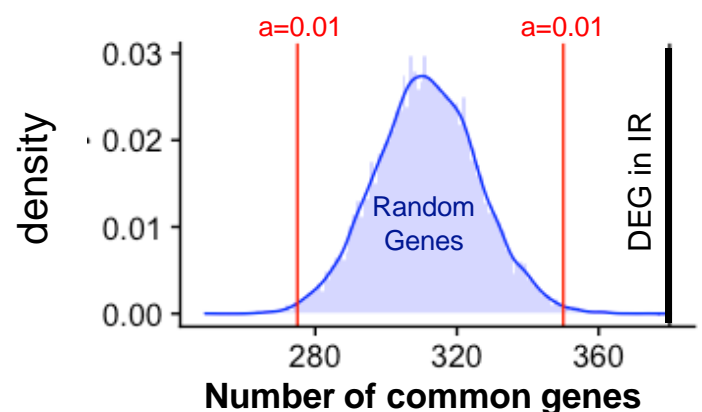

E.

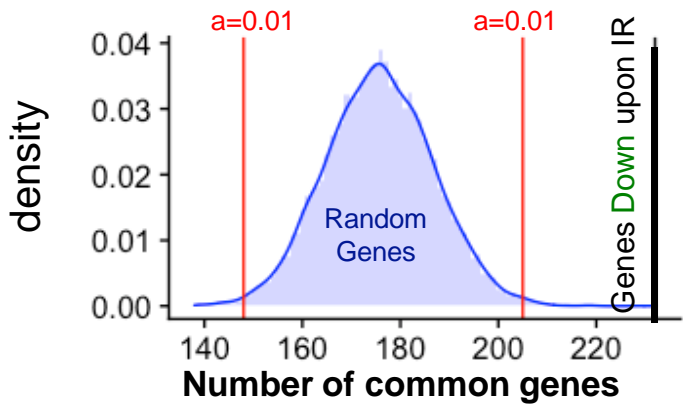

Number of common genes
D.

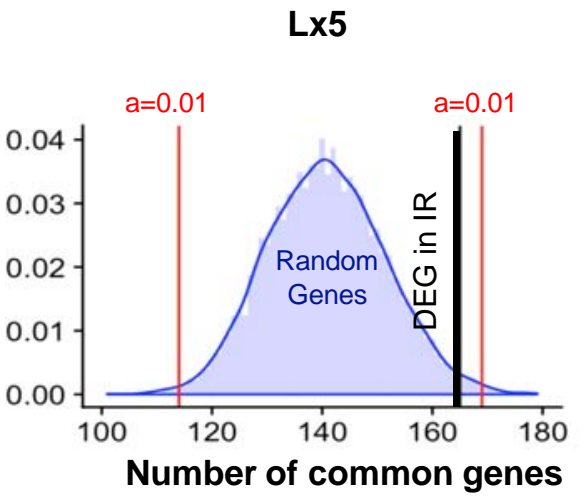

F.

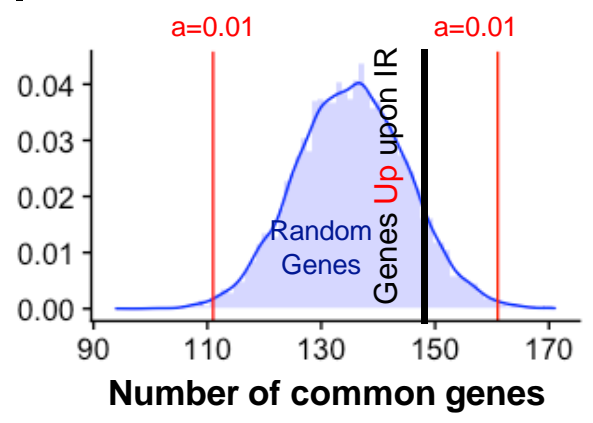

Number of common genes

Genes Up

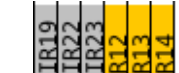

242 SampleName

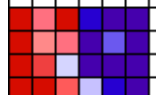
Dnajb4 Dock1 Prkcdbp Rab12

Akt3

Rcor2 Slc6a15 Kdelc2 Meis1 Zfp24 Ptrf Myo5 Eya2 Sesn1 Pkia Rarb $1700029707 \mathrm{z}$ 170002 Zfp780 Mras Ptger3 Gramd1c Lrrc1 Tcea2 Prdm5 Wfdc2

4933431 E20R $\mathrm{Ghr}$ Pcmtd2 Vmn2r34 Stox 1

$\mathrm{Nap} 113$

Arhgap

Arnga

Istr

Tnfsfg

Ifp462

Ifih1

Sorbs

Jam3

Pomt2

Ddx19a

Fads 1

Ttc8

Srgap

Myof

$\operatorname{cog} 6$

$\mathrm{Xk}$

Mapre3

Rora

Zfp37

Gm973

Thtpa

Med23

Spire1

Zkscan

Rex2

Ccdc91

Ift 122

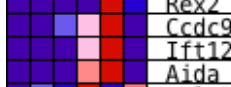

Aida
Polr2b
Caplo

Capsl

Plekha

Fpgt

Slc41a1

Tceal5

Zfp 22

Copb1

Atad1

Cat 
Figure 4. Gene repression upon IR is associated with intronic L1Md. (A) Correlation plot representing the $\log 2$ (Fold change) in $\mathrm{H} 3 \mathrm{~K} 9 \mathrm{me} 3$ concentration at gene promoters $v s \log 2$ (Fold change) in gene expression. (B-F) Permutation test comparing of the number of genes found in common between the list of DEG or 10000 lists of random genes and the list of genes hosting one or several L1Md. Blue curve: Distribution of the number of genes found in common between random genes and genes hosting an L1. Black vertical line: number of genes found in common between DEG and genes hosting an L1Md (C) or an Lx5 (D); or between genes downregulated (E) or upregulated (F) upon IR and hosting an L1Md. Significance bars $(p<0.01)$ are shown in red. (H-I) Heatmaps of the expression of genes from two LT-HSC signatures that are significantly up(red) or down- (blue) regulated in IR vs NIR. Green stars indicate the presence of an intronic L1Md in the downregulated gene. 


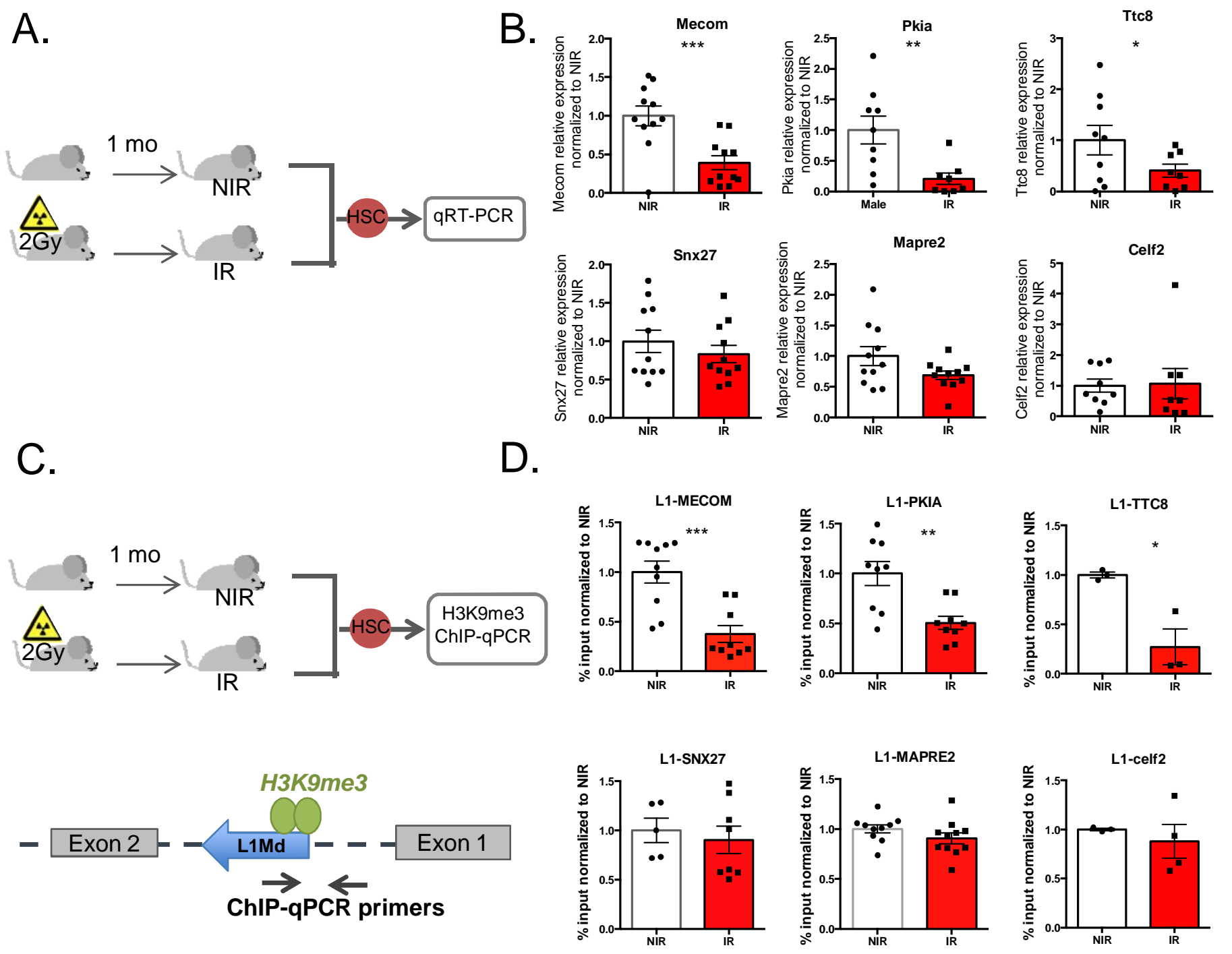

E.

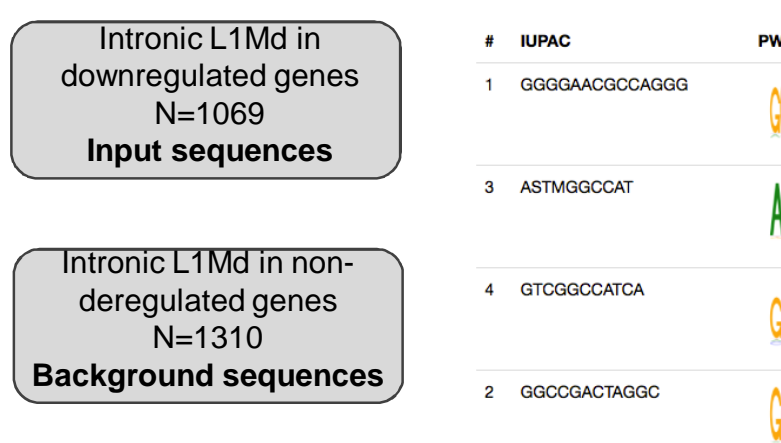

\section{F.}

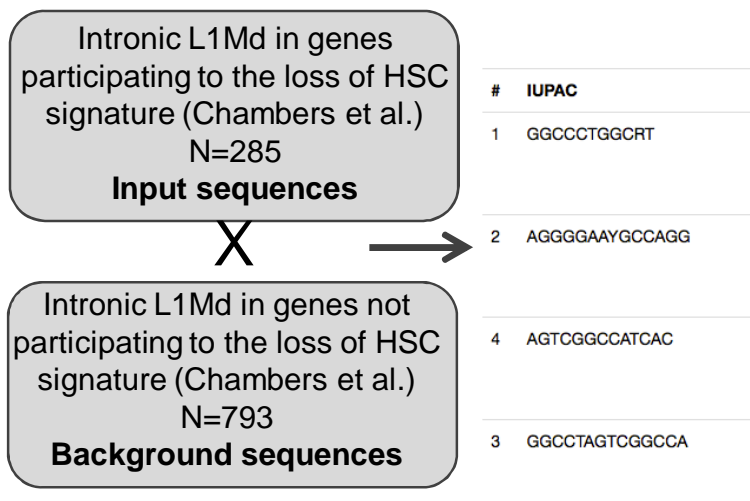


Figure 5. Gene repression upon IR is associated with loss of H3K9me3 at intronic L1Md loci harboring NF-kB binding sites. (A, B) Experimental design and mRNA expression assessed by RT-qPCR in HSCs one month post TBI. Ct values were normalized to RPL32 and HPRT. Results are expressed as fold change from the mean value of the NIR condition. Each dot represents a pool of 3 (NIR) or 4 (IR) mice. Means +/- SEM from 3 to 4 independent experiments. (C, D) Experimental design and H3K9me3 ChIP-qPCR enrichment 1 month post TBI. The primer positioning at the intronic L1Md allowing the amplification of a unique and specific product is shown (C). Each dot represents a pool of 3 (NIR) or 4 (IR) mice from 2-4 independent experiments. Results are means $+/-$ SEM of the percentage of input normalized to the NIR condition. *** $\mathrm{p}<0.001$; $* *<<0.01$; $* \mathrm{p}<0.05$ t-test. (E-F) De novo motif discovery analysis performed with the BaMMmotif tool on L1Md sequences located in introns of downregulated genes $v s$ non-deregulated genes (E) or the genes participating vs not participating to the loss of the LT-HSC signature (F). Enriched motifs were matched to known motifs using the Hocomoco mouse database. 


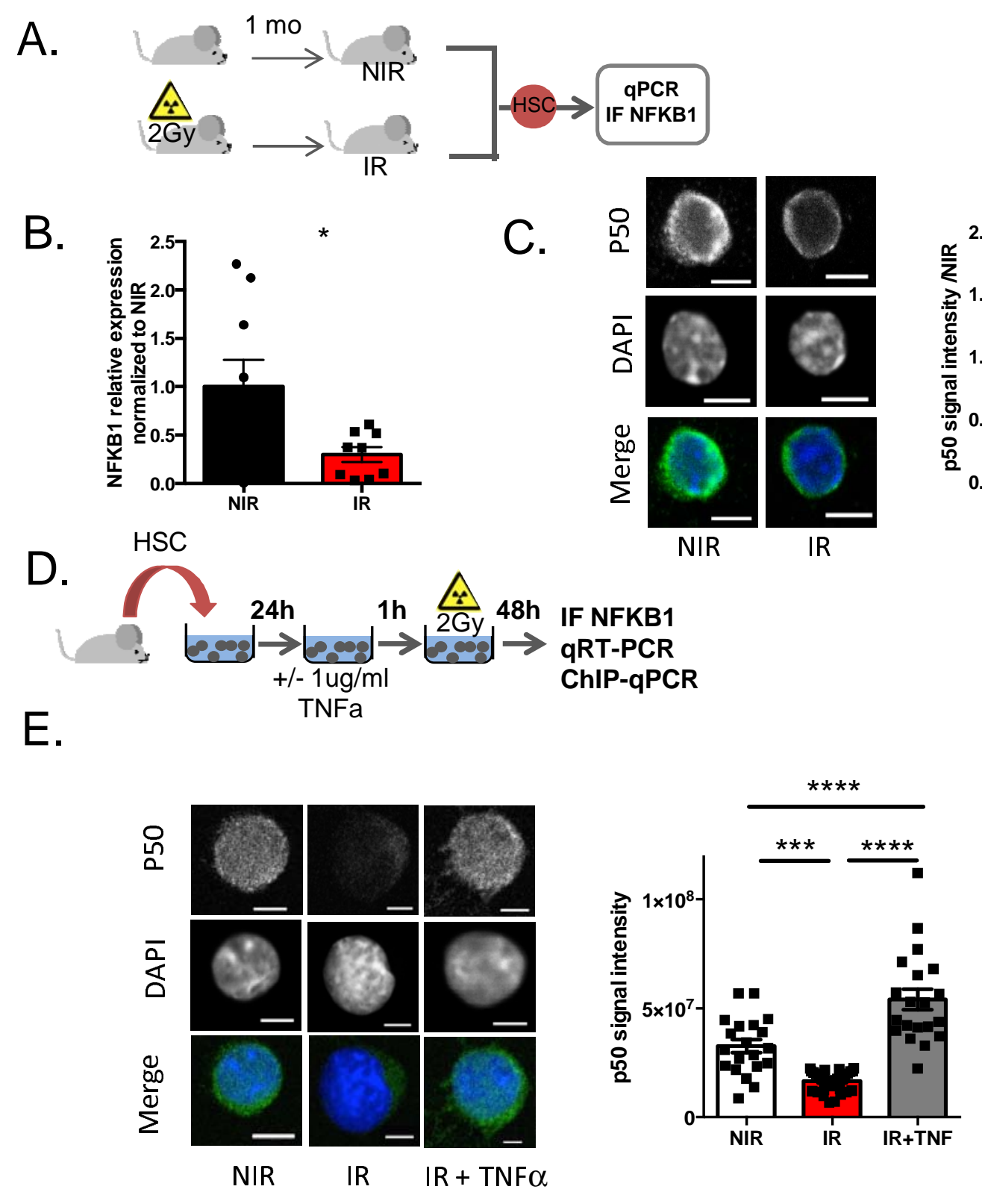

F.
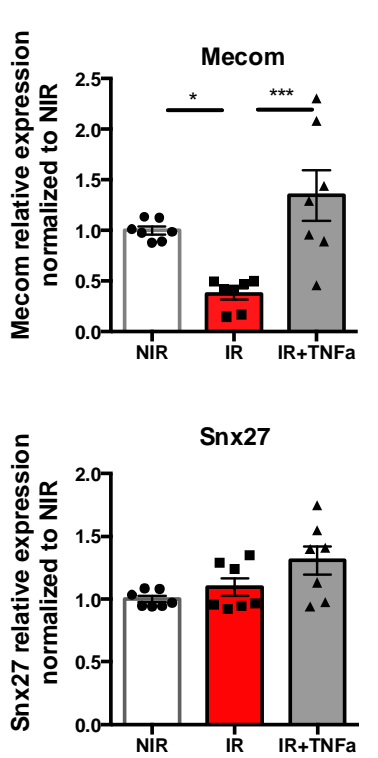
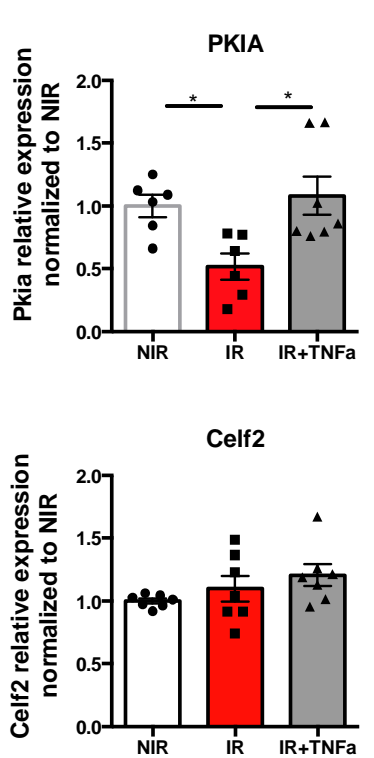

$G$
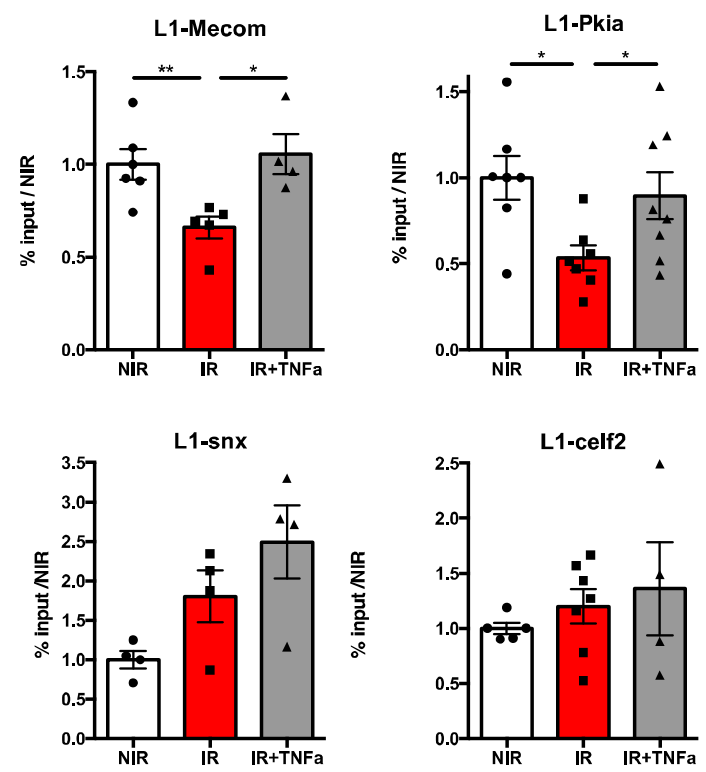
$\mathrm{H}$.
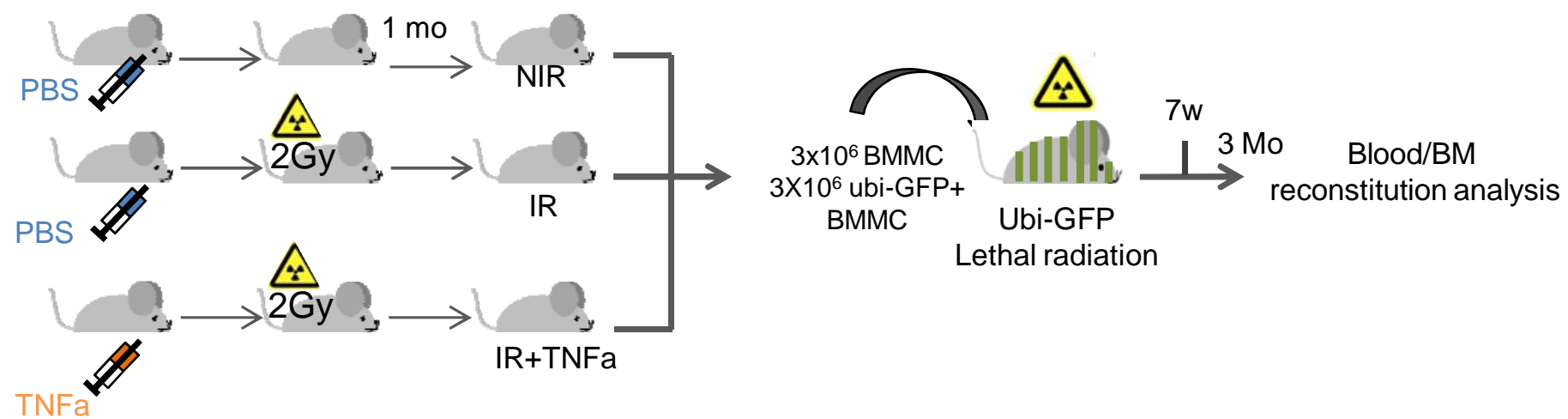

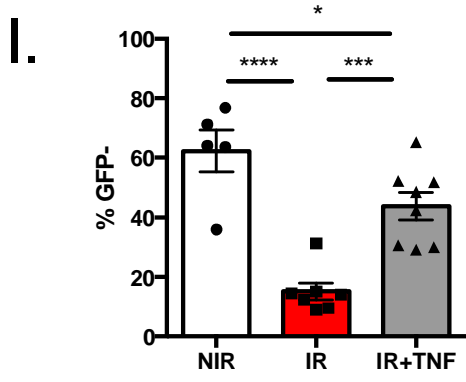

J.

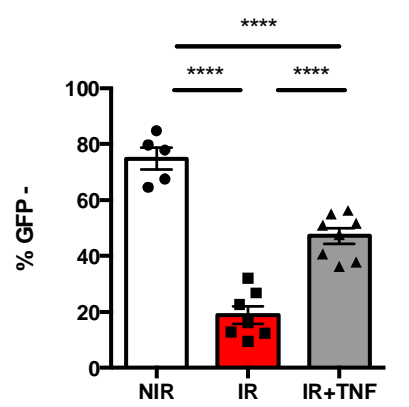

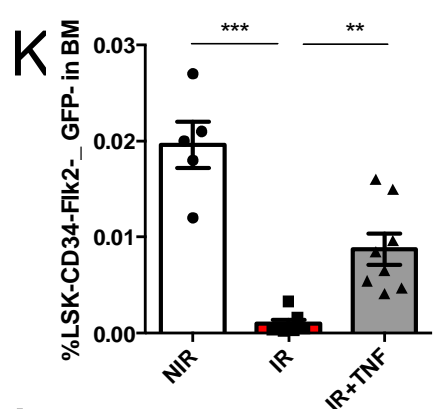

L.

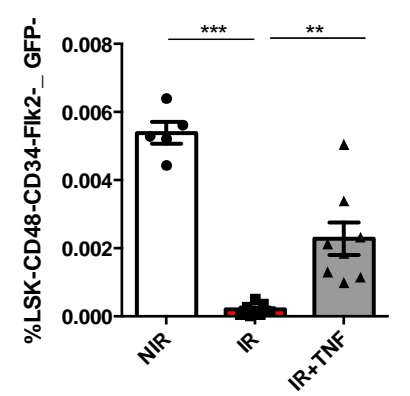

M.

\section{Non-irradiated}

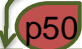

TNF $\alpha$

p50 p50

RNAPol II

Exon 2

H31. HMT 50

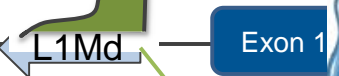

Irradiated

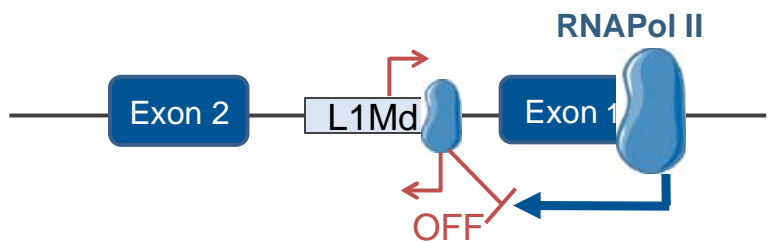


Figure 6. TNF-a treatment prevents loss of $\mathrm{H3H9me3}$ at intronic L1Md,HSC gene repression and HSC loss of function. (A-C) NFKB1 expression in HSCs one month after TBI. (B) mRNA expression measured by RT-qPCR. Ct values were normalized to mean of RPL32 and HPRT. Results are expressed as fold change from the mean value of the NIR condition. Means +/- SEM. Each dot represents a pool of 3 (NIR) or 4 (IR) mice from 3 independent experiments. * ${ }^{*}<0.05$ t-test. (C) Representative images and quantification of Nfkb1 protein mean IF intensity. Bars, $5 \mu \mathrm{M}$. Each dot represents a cell. Results are expressed as fold change from the mean value of the NIR condition from 2 independent experiments and represented as means $+/-$ SEM.*** $\mathrm{p}<0.001 \mathrm{t}$-test. (D-G) Experimental design analyzing the effects of IR and TNF-a in vitro. (E) Representative images and quantification of Nfkb1 staining. Bars, $5 \mu \mathrm{M}$. Each dot represents a cell. Results are represented as mean +/- SEM of Nfkb1 IF intensity. One-way ANOVA with Tukey's multiple comparison test. (F) Gene expression evaluated by RT-qPCR. Means +/- SEM from 2 independent experiments. One-way ANOVA with Tukey's multiple comparison test. (G) H3K9me3 enrichment at intronic L1Md evaluated by ChIP-qPCR. Results are expressed as in legend to Figure 5B. Means +/- SEM from 2-3 independent experiments. One-way ANOVA with Bonferroni's multiple comparison test.

(H) Experimental design for reconstitution experiments using HSC sorted from mice one month after TBI and previously treated with TNF- $\square$ (IR+TNF) or not (IR), or left untreated (NIR); BMMC, bone marrow mononuclear cells. (I-J) percentage of GFP-negative donor contribution in blood in mice transplanted with NIR, IR or IR+TNF cells at 7 (I) and 14 weeks (J) after reconstitution. (K, L) LSK CD34-Flk2-GFP- (K) or LSK CD34-Flk2-CD48-(L) GFP-negative donor HSC contribution in the BM 14 weeks after reconstitution. One-way ANOVA Tukey's multiple comparison test. *p<0.05; $* * \mathrm{p}<0.01$; *** $\mathrm{p}<0.001 ; * * * * \mathrm{p}<0.0001$. (F) Model. At basal, the NF- $\square \mathrm{B}$ pathway, possibly through its repressor NFKB1 (p50/p50 homodimers), is involved in the recruitment of H3K9 methylases (HMT) at intronic L1Md enriched in NFKB binding sites motifs, and apposition of the repressive histone mark H3K9me3. H3K9me3 "islands" into the body of transcribed genes may help the processing of RNAPolymerase II (RNAPolII) and transcript stability. Upon Irradiation, loss of the TNF- $\square$-NF- $\square$ B pathway leads to a loss of $\mathrm{H} 3 \mathrm{~K} 9 \mathrm{me} 3$ at the intronic L1Md, gene repression and transcript stability. Alternatively, loss of $\mathrm{H} 3 \mathrm{~K} 9 \mathrm{me} 3$ at intronic L1Md may lead to their transcription and gene repression through transcriptional interference. 